\title{
Large-Scale Fluid-Structure Interaction Simulation of Viscoplastic and Fracturing Thin-Shells Subjected to Shocks and Detonations
}

\author{
Fehmi Cirak ${ }^{*}$, Ralf Deiterding ${ }^{\dagger}$, and Sean P. Mauch ${ }^{\ddagger}$ \\ *University of Cambridge, Department of Engineering \\ Cambridge, CB2 1PZ, UK \\ e-mail: fc286@eng.cam.ac.uk \\ †Oak Ridge National Laboratory \\ P.O. Box 2008 MS 6367, Oak Ridge, TN 37831, USA \\ e-mail:deiterdingr@ornl.gov \\ ${ }^{*}$ California Institute of Technology, \\ Mail Code 158-79, Pasadena, CA 91125, USA \\ e-mail: sean@cacr.caltech.edu
}

Key words: Fluid-structure interaction, thin-shells, detonations, water hammer, large deformations, fracture, dynamic mesh adaptation, parallelization

\begin{abstract}
The fluid-structure interaction simulation of shock- and detonation-loaded thin-walled structures requires numerical methods that can cope with large deformations as well as local topology changes. We present a robust level-set-based approach that integrates a Lagrangian thin-shell finite element solver with fracture and fragmentation capabilities and an Eulerian Cartesian fluid solver with optional dynamic mesh adaptation. As computational applications, we consider the plastic deformation of a copper plate impacted by a strong piston-induced pressure wave inside a water pipe and the induction of large plastic deformations and rupture of thin aluminum tubes due to the passage of ethylene-oxygen detonations.
\end{abstract}

\section{Introduction}

The Center for Simulation of Dynamic Response of Materials at the California Institute of Technology has developed a novel virtual test facility (VTF) framework for studying the three-dimensional dynamic response of solid materials subjected to strong waves propagating in compressible fluids $[1,30,17,40]$. The VTF targets inherently coupled problems, such as the transient deformation of metallic structures due to the explosive detonations or the fracture and fragmentation of brittle or ductile materials under shock wave impact. This application regime requires the coupled utilization of computational fluid dynamics (CFD) solvers for compressible hydrodynamics and computational solid dynamics (CSD) solvers for large plastic material deformations. CFD and CSD solvers both need to be time-accurate and 
have to consider all arising supersonic wave phenomena (e.g., shear and dilatation waves in the plastic solid, shock waves in the compressible fluid) correctly. Applicable numerical schemes are usually shock-capturing and time-explicit. For coupling, we therefore employ a temporal splitting technique in which CFD and CSD solver exchange data only at the interface between disjoint computational domains after consecutive time steps. For compressible fluids, stable solutions are obtained reliably with such a "weakly coupled" method, when the evolving interface geometry and velocities are imposed as boundary conditions on the CFD solver and the hydrodynamic pressure is used as force boundary condition acting on the solid exterior $[38,3,26,11]$.

While a Lagrangian representation is most suitable to account numerically for large solid deformations, contact and fracture, shock-capturing methods for compressible flows are most easily formulated in an Eulerian frame of reference. The idea behind the VTF is to develop a fluid-structure interaction (FSI) framework, including fluid mesh adaptation, that supports aforementioned coupling methodology and allows the embedding of propagating triangulated surface meshes derived from an arbitrary CSD solver into easily exchangeable Cartesian Eulerian CFD solvers. This concept facilitates solver re-use and modularization (see also [27] for further discussion of modular concepts for FSI simulation). Specific to the VTF is that scalar level set functions storing the distance information to the embedded surface are used to represent the complex geometry on the Cartesian fluid mesh and a ghostfluid-type approach is employed to impose fluid boundary conditions $[20,19,3]$.

In the present paper, we extend the VTF to the computation of plastically deforming and fracturing thin-shells subjected to water shocks and gaseous detonations. This extension includes equation of state models for liquids and detonations, viscoplastic finite deformation thin-shell elements, thin-shell fracture modeling with cohesive interfaces, and robust algorithmic treatment of fluid-structure interaction in presence of fracture and fragmentation. For validation and verification of the presented framework we consider two experiments: First, the plastic bulging of a thin copper plate sealing the end of a water pipe when a piston at the opposite end is struck by a steel projectile. Second, venting and rupture simulations of thinwalled aluminum tubesdue to the passage of an internal gaseous ethylene-oxygen detonation [7]. The latter studies are motivated by accidents in cooling systems of power-plants and are intended to serve as a large-scale multi-physics validation case for the VTF.

In Sec. 2, we sketch the adaptive Cartesian finite volume fluid solver with levelset-based embedded boundary capability and detail the employed models for water and detonation wave simulation. Section 3 describes the CSD solver that has been developed to enable FSI simulations of thin-walled (possibly fracturing) shell structures. The solver is founded on a Kirchhoff-Love type thin-shell formulation in Lagrangian coordinates and achieves a consistent finite element discretization of the 
underlying energy functional even in the case of fracture by employing subdivision elements. In Sec. 4, we outline the highly efficient auxiliary algorithm based on geometric characteristic reconstruction and scan conversion that we have developed to transform evolving triangulated surface meshes efficiently into signed or unsigned distance functions. The fluid-structure coupling algorithm, highlighting particular its incorporation into the adaptive fluid mesh refinement framework and the implementation on distributed memory computing platforms is detailed in Sec. 5. In Sec. 6, we present a FSI simulation of a plastically deforming circular thin copper plate under water hammer that appears to be in very good agreement with experimental and analytic results. Simplicity of the setup and given level of detail make for an excellent verification testcase for shock-driven fluid-structure interaction. The parallel performance of our FSI code and the savings from utilizing dynamic mesh adaptation in the fluid are also discussed. In Sec. 7, we describe our efforts in simulating detonation-driven tube rupture events focusing particularly on the verification and validation of the fluid sub-problem that involves detonation modeling. We present one large-scale FSI computation in the plastically deforming regime in which the longitudinal opening is simplified by cutting two identical flaps previously into the tube that is found to be in qualitative agreement with experimental observations. The final FSI computation is a test simulation of a detonation-driven tube fracture event that demonstrates versatility, robustness and relevancy of the proposed level-set-based coupling approach for FSI problems with complex geometry evolutions.

\section{Eulerian fluid mechanics solver}

The simulation of strong trans- or supersonic wave phenomena in fluids requires the consideration of the compressibility while viscosity can typically be neglected. The basic system of governing equations are the Euler equations:

$$
\begin{array}{ccc}
\partial_{t} \rho+ & \nabla \cdot(\rho \boldsymbol{u}) & =0, \\
\partial_{t}(\rho \boldsymbol{u})+ & \nabla \cdot(\rho \boldsymbol{u} \otimes \boldsymbol{u})+\nabla p & =0 \\
\partial_{t}(\rho E)+ & \nabla \cdot((\rho E+p) \boldsymbol{u}) & =0
\end{array}
$$

Herein, $\rho$ is the density, $\boldsymbol{u}$ the velocity vector and $E$ the specific total energy. In order to close $(1)$, an equation of state $p=p(\rho, e)$ is required for modeling the dependency of the hydrostatic pressure $p$ on density $\rho$ and specific internal energy $e:=E-\frac{1}{2} \boldsymbol{u}^{T} \boldsymbol{u}$. For a single polytropic gas, the equation of state reads

$$
p=(\gamma-1) \rho e
$$

with $\gamma$ denoting the constant adiabatic exponent. For Eq. (2), the speed of sound in the fluid $c$ is found to be $c=(\gamma p / \rho)^{1 / 2}$. Equations (1) plus Eq. (2) can be shown to 
be a nonlinear system of conservation laws of hyperbolic type that admits discontinuous solutions, involving shocks and contact discontinuities, even from smooth initial data. The standard approach in computing discontinuous solutions of (1) numerically is to use a shock-capturing Eulerian finite volume discretization with a flux approximation that achieves proper local upwinding in all characteristic fields. Except for stationary problems, such methods are always time-explicit. Several ex-

cellent textbook descriptions are nowadays available for frequently used schemes for (1) plus (2) and we will refer to the literature where appropriate.

\subsection{Modeling pressure waves in liquids}

The propagation of pressure waves in liquids can be modeled with good accuracy with the inviscid Euler equations. For very high pressures, as they appear for instance in underwater explosions, a simple extension of Eq. (2) to the "stiffened" gas equation of state of the form

$$
p=(\gamma-1) \rho e-\gamma p_{\infty}
$$

presents a viable model. For the equation of state (3), the speed of sound reads

$$
c=\left(\gamma \frac{p+p_{\infty}}{\rho}\right)^{1 / 2} .
$$

When both $\gamma$ and $p_{\infty}$ are assumed to be constant, the partial derivatives of $p$ with respect to $\rho$ and $e$ remain unaltered and numerical methods derived for Euler equations and the polytropic gas equation (2) can be trivially extended to the stiffened case by simply replacing all pressure and speed of sound evaluations by (3) and (4), respectively. The simulations presented in Sec. 6 use the approximative Riemann solver of Roe with the maximally second-order-accurate fully multi-dimensional Wave Propagation method of LeVeque. See [24] for details.

\subsection{Modeling gaseous detonation waves}

A detonation is a supersonic shock-induced combustion wave that consists of a discontinuous hydrodynamic shock followed by smooth region of chemical reaction toward the equilibrium state. For premixed gaseous combustion, the model of the inviscid Euler equations is generally accepted [21]. In here, we consider only the simplified case of a single exothermic chemical reaction $A \longrightarrow B$ with a progress variable $Y$ corresponding to the mass fraction ratio between the partial density of the reactant $A$ and the total density $\rho$, i.e. $Y=\rho_{\mathrm{A}} / \rho$. The reaction is incorporated into the Euler equations by extending (1) with the additional inhomogeneous conservation law

$$
\partial_{t}(Y \rho)+\nabla \cdot(Y \rho \boldsymbol{u})=\psi
$$


and by utilizing the equation of state $p=(\gamma-1)(\rho e-\rho Y q)$ instead of (2). In the latter, the parameter $q$ denotes the heat release due to the chemical reaction per unit mass, which determines the detonation speed uniquely, cf. [21, 15]. A one-step reaction would typically be modeled with an Arrhenius law such as [21]

$$
\psi=-k Y \rho \exp \left(\frac{-E_{\mathrm{A}} \rho}{p}\right),
$$

but in the specific case considered here, we utilize the constant volume burn model suggested by Mader [28]. This model neglects the detailed chemical depletion, and therefore the internal detonation structure, but ensures the right propagation speed and the correct state in chemical equilibrium at all grid resolutions. The model is intended to be applied together with the fractional step method that numerically decouples chemical reaction and hydrodynamic transport. First, the homogeneous system $(1),(5)$ is advanced at a full time step, then the reactant density $\rho_{\mathrm{A}}$, pressure $p$, and total energy $E$ are modified locally in each cell; the total density $\rho$ and the velocity vector $\boldsymbol{u}$ remain unaltered. The algorithm for the detonation model reads:

$$
\begin{aligned}
& V:=\rho^{-1}, V_{0}:=\rho_{0}^{-1}, V_{\mathrm{CJ}}:=\rho_{\mathrm{CJ}} \\
& Y^{\prime}:=1-\left(V-V_{0}\right) /\left(V_{\mathrm{CJ}}-V_{0}\right) \\
& \text { if } 0 \leq Y^{\prime} \leq 1 \text { and } Y>10^{-8} \\
& \quad \text { if } Y<Y^{\prime} \text { and } Y^{\prime}<0.9 \text { then } Y^{\prime}:=0 \\
& \quad \text { if } Y^{\prime}<0.99 \text { then } p^{\prime}:=\left(1-Y^{\prime}\right) p_{\mathrm{CJ}} \text { else } p^{\prime}:=p \\
& \quad \rho_{\mathrm{A}}:=Y^{\prime} \rho, E:=p^{\prime} /(\rho(\gamma-1))+Y^{\prime} q+\frac{1}{2} \boldsymbol{u}^{T} \boldsymbol{u}
\end{aligned}
$$

In the latter, the index 0 indicates the unreacted state (assumed to be constant), while CJ refers to the equilibrium values that can be calculated in advance for a given detonation velocity following Chapman-Jouguet theory [21, 15].

For all simulations involving the detonation model, we utilize a straightforward, but non-trivial, extension of the flux-vector splitting (FSV) method by Van Leer (cf. [15]). The Van Leer FSV is more diffusive than Roe's approximate Riemann solver, but can be proven to be positivity-preserving and is significantly more robust to numerical alterations in the vector of state by the detonation model and our embedded boundary incorporation technique. When using Van Leer FVS, we achieve secondorder accuracy in smooth solution regions by employing the MUSCL-Hancock variable extrapolation technique and use dimensional splitting for the three-dimensional extension. Detailed accounts of both techniques can be found in [39].

\subsection{Numerical treatment of thin-walled structures}

In the Virtual Test Facility, we follow a ghost-fluid-type approach to incorporate geometrically complex moving boundaries scheme-independent into originally Cartesian 


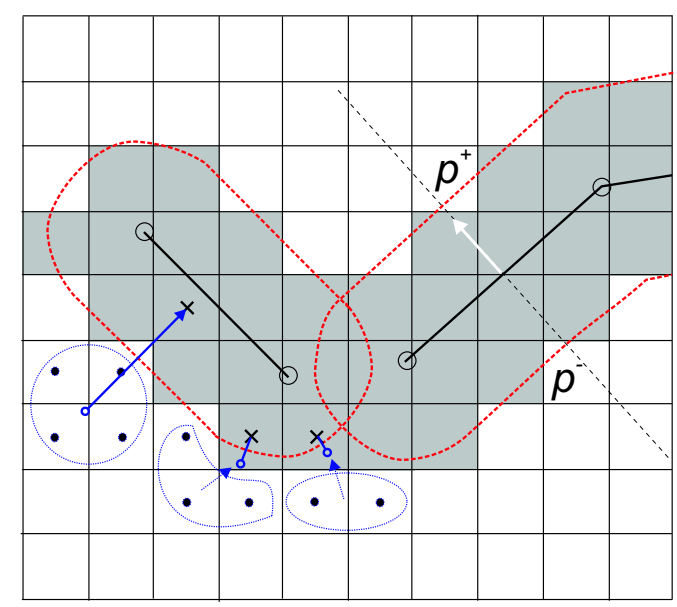

Figure 1: Ghost cells (shaded gray) around shell elements (dark segments) and construction of mirrored values.

upwind discretization. The idea of this approach is to use some of the finite volume cells as ghost cells for enforcing immersed moving wall boundary conditions [20]. The boundary geometry is mapped onto the Cartesian mesh by employing a scalar level set function $\phi$ that stores the distance to the boundary surface and allows the efficient evaluation of the boundary outer normal in every mesh point as $\boldsymbol{n}=-\nabla \phi /|\nabla \phi|$. A cell is considered to be a valid fluid cell, if the distance at the cell midpoint satisfies the condition $\phi>h / 2$, where $h$ is the shell thickness, and as an exterior ghost cell otherwise. The mesh received from the shell solver corresponds to a two-dimensional oriented manifold surface mesh (cf. Sec. 3) that, in the most general case, can be arbitrarily surrounded by fluid. Utilizing condition $\phi>h / 2$ is a straightforward, unambiguous solution to achieve the mandatory thickening of this mesh by the shell thickness $h$. The contour line $\phi=h / 2$ effectively represents the embedded boundary for the fluid solver (depicted as dotted line around shell elements in Fig. 1). The hydrodynamic load on each shell element is evaluated as the difference between the approximated pressure values at $\phi=h / 2$ in the positive and negative direction of each element's normal, i.e. $p^{F}:=p^{+}-p^{-}$. While in the general case of arbitrary topology evolutions only unsigned distance can be used, closed surfaces also allow the utilization of signed distance. In this paper, signed distance was only used for the FSI simulation of Sec. 6 .

For the Euler equations, the boundary condition at a rigid wall moving with velocity $\boldsymbol{w}$ is $\boldsymbol{u} \cdot \boldsymbol{n}=\boldsymbol{w} \cdot \boldsymbol{n}$. Enforcing the latter with ghost cells, in which the discrete values are located at the cell centers, requires the mirroring of the primitive values $\rho$, $\boldsymbol{u}, p$ and $\rho_{\mathrm{A}}$, when applicable, across the embedded boundary. The normal velocity in the ghost cells is set to $(2 \boldsymbol{w} \cdot \boldsymbol{n}-\boldsymbol{u} \cdot \boldsymbol{n}) \boldsymbol{n}$, while the mirrored tangential velocity remains unmodified. Mirrored values are constructed by calculating spatially interpolated 
values in the point $\tilde{\boldsymbol{x}}=\boldsymbol{x}+2 \phi \boldsymbol{n}$ from neighboring interior cells. We employ a dimension-wise linear interpolation for this operation, but it has to be emphasized that directly near the boundary the number of interpolants needs to be decreased to ensure the monotonicity of the numerical solution. This property is essential in simulating hyperbolic problems with discontinuities. Figure 1 also highlights the necessary reduction of the interpolation stencil for some exemplary cases. The interpolation locations are indicated by the origins of the arrows normal to the contour line that defines the embedded boundary. After the application of the numerical scheme, cells that have been used to impose internal boundary conditions are set to the entire state vector of the nearest cell in the fluid interior. This operation ensures proper values in case such a cell becomes a regular interior cell in the next step due to boundary movement. The consideration of $\boldsymbol{w}$ in the ghost cells guarantees that the embedded boundary propagates at most one cell in every time step.

Note that the described technique does not require a modification of the numerical stencil itself and is therefore generically applicable, but causes a diffusion of the boundary location throughout the method and results in an overall non-conservative scheme. We alleviate such errors and the unavoidable staircase approximation of the boundary with this approach effectively by using the dynamic mesh adaptation technique described in the next sub-section to also refine the Cartesian mesh appropriately along the boundary. Some authors have also presented cut-cell techniques that utilize the correct boundary flux. However, the proposed numerical circumventions of the severe time step restriction in time-explicit schemes [37, 5], which can result from small cells created by the boundary intersection, are logically quite complicated. Most approaches have not yet been extended successfully to three spatial dimensions even for pure fluid flow problems.

\subsection{Structured adaptive mesh refinement}

In order to supply a fine local temporal and spatial resolution efficiently, the finite volume scheme described above has been incorporated into the block-structured adaptive mesh refinement (SAMR) method after Berger and Colella [4]. Characteristic for the idea of structured mesh adaptation is that the finite volume method is technically not implemented in a cell-based fashion but rather in a routine which operates on equidistant subgrids. The subgrids become computationally decoupled during one update through the use of ghost or halo cells. Cells being flagged for refinement (shaded in Fig. 2) are clustered recursively into non-overlapping rectangular subgrids and a hierarchy of successively embedded levels is thereby constructed (cf. Fig. 2). Starting from the base mesh on level 0, the time step size and all spatial mesh widths on level $l>0$ are $r_{l}$-times finer than on level $l-1$ and a time-explicit finite volume scheme will (in principle) remain stable on all levels of the hierarchy. 


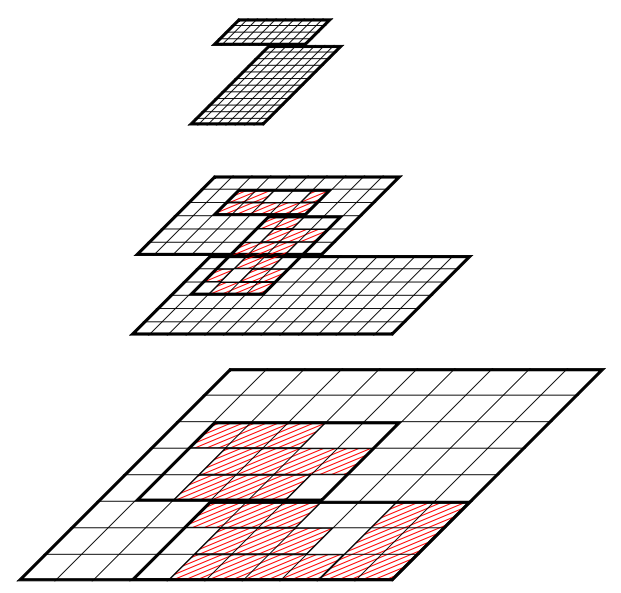

Figure 2: SAMR hierarchy.

Different levels are integrated recursively in time allowing the derivation of temporally and spatially interpolated boundary conditions of Dirichlet-type from the coarser level at coarse-fine interfaces. Values of cells covered by finer subgrids are overwritten by averaged fine grid values subsequently. This operation leads to a modification of the numerical stencil on the coarse mesh and requires a special flux correction in cells abutting a fine grid. In order to ensure discrete conservation (at least for purely Cartesian problems without embedded boundaries), but particular to enforce a von Neumann-type boundary condition matching at coarse-fine interfaces subsequently, the coarse flux approximation adjacent to modified coarse level cells is replaced with the sum of all overlying fine level fluxes. See [4] or [15] for details.

SAMR in the VTF is provided generically by the AMROC (Adaptive Mesh Refinement in Object-oriented $\mathrm{C}++$ ) framework [14]. AMROC has been parallelized effectively for distributed memory machines [16] and can be used on all systems that provide the MPI library. The parallelization strategy is a rigorous domain decomposition approach that partitions the SAMR hierarchy from the root level on. The key idea is that all higher level domains are required to follow this "floor plan". In AMROC, a generalization of Hilbert's space-filling curve [34] is currently used to derive load-balanced root level distributions at run time.

\section{Lagrangian thin-shell solver}

The used Kirchhoff-Love type thin-shell model has been discretized with smooth subdivision finite elements, as previously introduced in $[8,9]$. Notably, the underlying kinematic assumptions allow for finite strains, displacements and rotations. The subdivision shell elements have also been extended to the range of applications that 


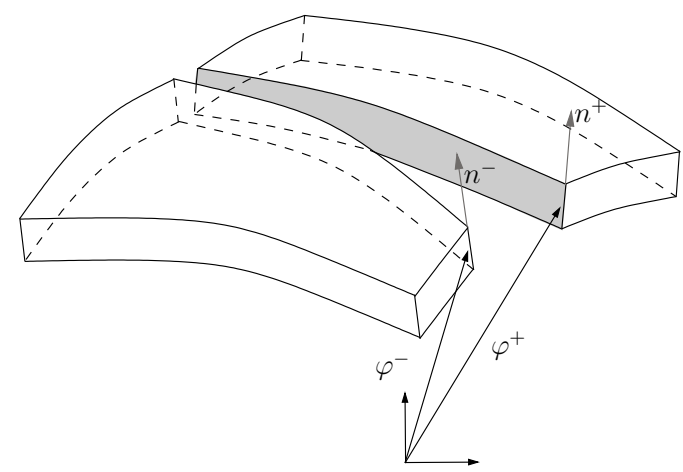

Figure 3: Fractured shell body: opposite crack flanks and corresponding normals.

involve fracture and fragmentation [10]. Thereby, fracture initiation and propagation is considered as a progressive failure phenomenon in which the separation of the crack flanks is modeled with a cohesive law. In the present implementation, cohesive interface elements are inserted at all inter-element edges and constrain the opening of the crack flanks to the deformation of the shell mid-surface and its normal. An alternative XFEM-based technique for computing shells with cracks was recently proposed by Areias et al. [2].

\subsection{Governing equations in weak form}

To kinematically describe a possibly fractured thin-shell as sketched in Fig. 3, we first consider the shell in its undeformed configuration $\bar{V}$. The position vector $\bar{\varphi}$ of a material point on the undeformed shell body is assumed to be

$$
\overline{\boldsymbol{\varphi}}=\overline{\boldsymbol{x}}+\theta^{3} \overline{\boldsymbol{n}}
$$

with the uniform thickness $\bar{h}$ and $-\bar{h} / 2 \leq \theta^{3} \leq \bar{h} / 2$. The position vector of the shell mid-surface is denoted by $\overline{\boldsymbol{x}}$ and its out-of-surface unit normal by $\overline{\boldsymbol{n}}$. In other words, the shell mid-surface represents a two-dimensional manifold in $\mathbb{R}^{3}$. The deformation mapping $\varphi$ maps the shell body into the deformed configuration $V$

$$
\boldsymbol{\varphi}=\boldsymbol{x}+\theta^{3} \lambda \boldsymbol{n}
$$

where $\boldsymbol{x}$ and $\boldsymbol{n}$ are the deformed mid-surface and its normal. The thickness stretch parameter $\lambda$ is the ratio of the deformed shell thickness $h$ to the reference thickness $\bar{h}$. In the presence of a crack, the deformation is discontinuous across the crack and has a jump, i.e.

$$
\llbracket \boldsymbol{\varphi} \rrbracket=\boldsymbol{\varphi}^{+}-\boldsymbol{\varphi}^{-}=\llbracket \boldsymbol{x} \rrbracket+\theta^{3} \llbracket \boldsymbol{n} \rrbracket
$$


where the superscripts + and - refer to the opposing crack flanks. Further, the first term describes the discontinuity of the deformation of the shell mid-surface, and the second term the discontinuity in the shell out-of-surface normal. The discontinuities in the deformations can also be interpreted as the opening displacement of the crack. Further, note that the Kirchhoff-Love constraint is satisfied, i.e. $\boldsymbol{x} \cdot \boldsymbol{n}=0$, on both sides of the crack.

A standard semi-inverse approach is followed for obtaining the shell equilibrium equations in weak from (see e.g. [6]). To this end, the assumed reduced kinematic equations for the shell body (Equations (7) and (9)) are introduced into the conventional virtual work expression for the three-dimensional body. As previously mentioned, we consider fracture as a gradual separation phenomenon, resisted by cohesive tractions. Consequently, the internal virtual work expression contains the virtual work of the cohesive interface $\left(\delta \Pi_{C \text {,int }}\right)$ in addition to the virtual work of the bulk material $\left(\delta \Pi_{S, \text { int }}\right)$

$$
\delta \Pi_{S, \text { int }}+\delta \Pi_{C, \text { int }}-\delta \Pi_{\text {ext }}=0
$$

with the external virtual work $\delta \Pi_{\text {ext }}$ and

$$
\delta \Pi_{S, \text { int }}=\int_{\bar{\Omega}} \int_{-\bar{h} / 2}^{\bar{h} / 2} \boldsymbol{P}: \delta \boldsymbol{F} \mu d \theta^{3} d \bar{\Omega}, \quad \delta \Pi_{C, \text { int }}=\int_{\bar{\Gamma}_{C}} \int_{-\bar{h} / 2}^{\bar{h} / 2} \boldsymbol{T} \cdot \llbracket \boldsymbol{\varphi} \rrbracket \mu d \theta^{3} d \bar{\Gamma}_{C},
$$

where $\boldsymbol{P}$ is the first Piola-Kirchhoff stress tensor, $\boldsymbol{T}$ the related traction vector at the cohesive surface, and $\boldsymbol{F}$ the deformation gradient. The virtual work expression for the bulk material is integrated over the undeformed shell mid-surface $\bar{\Omega}$ and for the cohesive interface over the crack path $\bar{\Gamma}_{C}$. The scalar factor $\mu$ accounts for the curvature of the shell in the volume computation [9]

\subsection{Subdivision thin-shell elements}

Next, we briefly outline the discretization of the governing equation (10) firstly for the non-fractured case. A detailed presentation of the used subdivision finite element discretization technique can be found in [8] and [9]. In this approach, the reference $(\overline{\boldsymbol{x}})$ and deformed $(\boldsymbol{x})$ shell surfaces are approximated using smooth subdivision surfaces belonging to the Sobolev space $H^{2}$ with square-integrable curvatures. The subdivision interpolation within one element is accomplished with shape functions, which have support on the element as well as on the one-ring of neighboring elements (Fig. 4(a)). The overlapping local subdivision interpolants, each defined over one patch, together lead to a global interpolant with square-integrable curvatures. Importantly, smoothness is achieved without introducing nodal rotations as degrees of freedom. The absence of nodal rotations is in particular in presence of finite rotations very appealing. The versatility of rotation-free shell elements has been studied by several authors [35, 32]. 


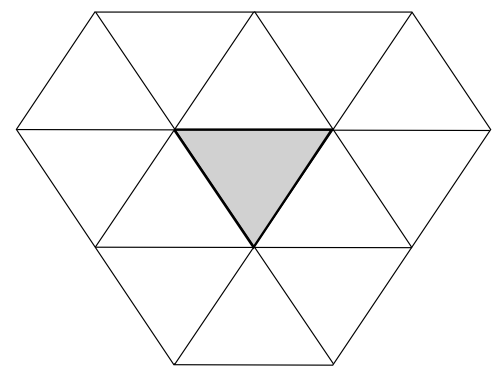

(a)

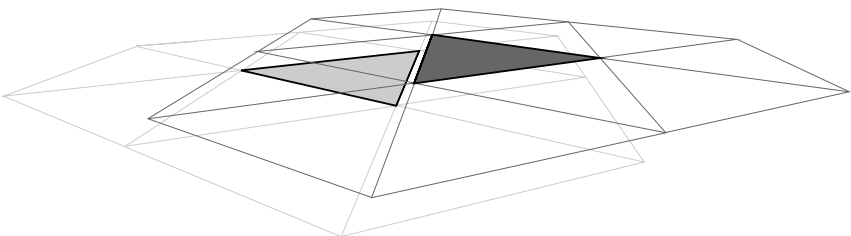

(b)

Figure 4: (a) A triangular subdivision element (shaded triangle at the center) and its 12 control nodes. (b) A cohesive edge and its two adjacent elements.

In the presence of fracture, the smoothness and/or continuity of the interpolation has to be relaxed and the subdivision interpolant needs to be modified (see [10] for details). The topological changes necessary to the non-local subdivision functions and the underlying control mesh in order to describe the dynamic propagation of a single crack are complicated. Therefore, we chose to pre-fracture the element patches, such that each patch possesses its own nodes and acts independently for the purpose of interpolation (see Fig. 4(b)). Prior to crack nucleation, the coupling of the elements is enforced by applying stiff elastic cohesive interfaces at all edges. Once fracture nucleates along an element edge, the element patches on both sides of the cracked edge interact through cohesive tractions. The cohesive tractions are self-balanced internal forces derived from a cohesive fracture model.

\subsection{Constitutive models for the shell}

An irreversible cohesive constitutive model as proposed by Ortiz et al. [33] is used for modeling the cracks. Thereby, the opening displacement $\llbracket \boldsymbol{\varphi} \rrbracket$ plays the role of a deformation measure while the traction $\boldsymbol{T}$ is the conjugate stress measure. Further, a scalar effective opening displacement is defined

$$
\delta=\sqrt{\beta^{2}\left|\boldsymbol{\delta}_{t}\right|^{2}+\left|\boldsymbol{\delta}_{n}\right|^{2}},
$$

where $\boldsymbol{\delta}_{t}$ and $\boldsymbol{\delta}_{n}$ are the tangential and normal displacement components of $\llbracket \boldsymbol{\varphi} \rrbracket$ with respect to the crack surface. The parameter $\beta$ assigns different weights to the tangential and normal opening displacements. The cohesive tractions $\boldsymbol{T}$ are given by

$$
\boldsymbol{T}=\frac{t}{\delta}\left(\beta^{2} \boldsymbol{\delta}_{t}+\boldsymbol{\delta}_{n}\right) .
$$




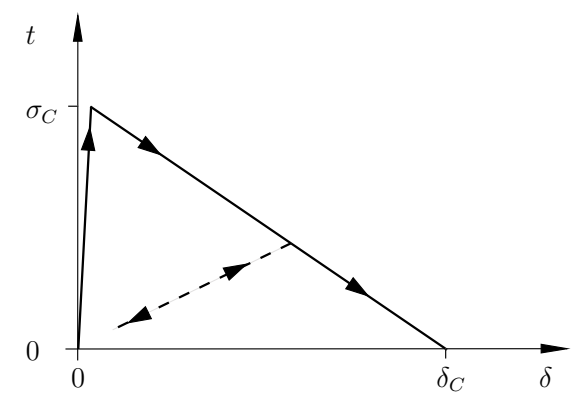

Figure 5: Irreversible linear cohesive law. Note that the initial stiff elastic response enforces the displacement continuity prior to crack initiation at $\sigma_{c}$. The dashed line represents the loading-unloading rule.

The scalar effective traction $t$ is computed from a cohesive law as shown in Fig. 5. In addition to the parameter $\beta$, the model parameters are the maximum tensile stress $\sigma_{c}$ and the critical opening displacement $\delta_{c}$. The following relationship between the cohesive law and the critical fracture energy $G_{c}$ exists:

$$
G_{c}=\int_{0}^{\infty} t d \delta
$$

which can be used for determining $\delta_{c}$. For further details see e.g. [33, 31].

The inelastic behavior of the bulk material, i.e. the relation between $\boldsymbol{P}$ and $\boldsymbol{F}$, is described with a conventional $J_{2}$ viscoplasticity model with isotropic power-law hardening as described in [12]. The power-law hardening for the flow stress $g$ has the form

$$
g\left(\epsilon^{p}\right)=\sigma_{y}\left(1+\frac{\epsilon^{p}}{\epsilon_{0}^{p}}\right)^{1 / n},
$$

where $\sigma_{y}$ is the initial yield stress, $\epsilon^{p}$ and $\epsilon_{0}^{p}$ are the total and the reference plastic strains, respectively, and $1 / n$ is the hardening exponent. The rate-dependent behavior is described in terms of the effective von Mises stress $\sigma_{\text {eff }}$ with a power viscosity law and constant rate sensitivity

$$
\sigma_{\mathrm{eff}}=g\left(\epsilon^{p}\right)\left(1+\frac{\dot{\epsilon}^{p}}{\dot{\epsilon}_{0}^{p}}\right)^{1 / m},
$$

where $\dot{\epsilon}_{0}^{p}$ is the reference plastic strain rate and $1 / m$ the strain rate sensitivity exponent.

The thin-shell typical plane stress condition is enforced with a local NewtonRaphson iteration at each quadrature point [13]. Thereby, the thickness stretch parameter $\lambda$ (Eq. 8) is the unknown variable in the iteration. 
(a)

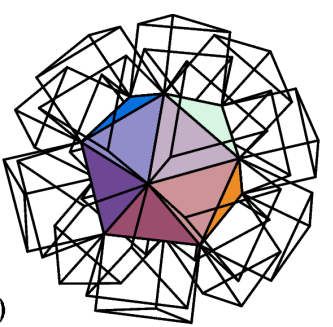

(b)

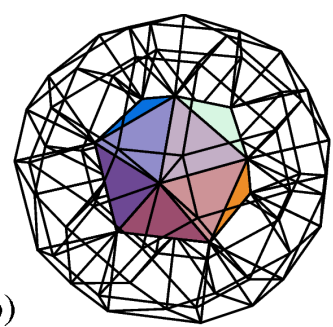

Figure 6: The characteristic polyhedra for faces and edges of an icosahedron.

\section{Efficient level set evaluation}

In Sec. 2, we have sketched the concept of employing a distance function to represent a complex embedded boundary on a Cartesian mesh. While distance functions are easily prescribed for single elementary geometric objects, their evaluation can be cumbersome for complex shapes. In coupled Eulerian-Lagrangian simulations, this complex shape is defined by the deforming shell surface mesh.

One can efficiently compute the distance on a grid by solving the eikonal equation with the method of characteristics and utilizing polyhedron scan conversion [29]. For a given fluid grid point, the relevant closest point on the triangular shell mesh lies on one of the mesh primitives face, edge or vertex. The characteristics emanating from each of these primitives form polyhedral shapes. Such a characteristic polyhedron contains all of the fluid grid points which are possibly closest to its corresponding face, edge or vertex. The closest points to a triangle face must lie within a triangular prism defined by the face and its normal; the closest points to an edge lie in a cylindrical wedge defined by the line segment and the normals to the two incident faces (see Fig. 6 for face (a) and edge (b) polyhedra for a particular example). Analogously, polygonal pyramids emanating from the vertices are also possible (not shown). We then determine the grid points that lie inside a characteristic polyhedron with polyhedron scan conversion. The polyhedron is first sliced along each sheet of the grid lattice to produce polygons, cf. Fig. 7. Simple geometric formulas are finally used to calculate the distance once a polyhedron has been assigned uniquely to each grid point.

By utilizing the outlined techniques, and evaluating the distance exactly only within a small distance around the surface, a highly efficient algorithm can be formulated that has linear computational complexity both in the number of Cartesian mesh points and the surface triangles [29, 17]. 

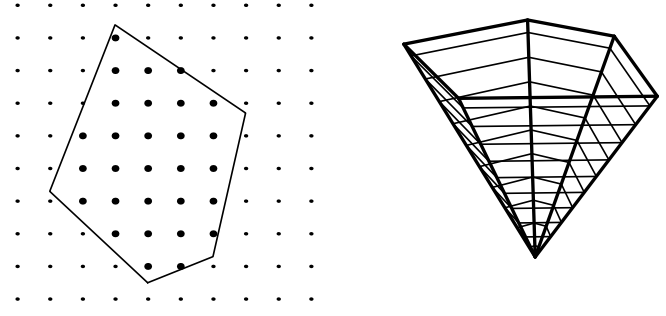

Figure 7: Scan conversion of a polygon in 2-D and slicing of a polyhedron to form polygons.

\section{$5 \quad$ Fluid-structure coupling}

The time-explicit fluid and solid solvers are weakly coupled by successively applying appropriate boundary conditions in a time-operator splitting technique. In the case of inviscid flows, the compatibility conditions are simply the continuity of the velocity component normal to the embedded boundary $u_{n}$ in solid $(\mathrm{S})$ and fluid $(\mathrm{F})$, i.e. $u_{n}^{S}=u_{n}^{F}$, and the continuity of the normal component of the solid's Cauchy traction vector, $p^{S}=(\boldsymbol{\sigma n}) \boldsymbol{n}$ with $\boldsymbol{\sigma}=1 / \operatorname{det}(\boldsymbol{F}) \boldsymbol{F P}$, and the hydrodynamic pressure $p^{F}$, i.e $p^{S}=p^{F}$. We use the following basic update algorithm to implement these coupling conditions numerically:

$$
\begin{aligned}
& \text { update } \phi(t) \\
& \boldsymbol{w}_{F}^{+/-}:=\boldsymbol{u}^{S}(t) \\
& \text { update_fluid }(\Delta t) \\
& p^{S}:=p^{F}(t+\Delta t) \\
& \text { update_solid }(\Delta t) \\
& t:=t+\Delta t
\end{aligned}
$$

After evaluating the distance function $\phi$ for the currently available shell surface mesh, the embedded wall boundary velocities for the fluid solver are set to the solid velocities in the nearest shell element mid-plane. The same velocity $\boldsymbol{w}$ is enforced in the fluid on upper $\left(^{+}\right)$and lower $\left(^{-}\right)$side of each element. After setting embedded rigid wall boundary conditions as sketched in Sec. 2 and the fluid update, a new hydrodynamic pressure load $p^{F}:=p^{+}-p^{-}$is computed, which is applied as an external pressure loading to the shell (compare Fig. 1). With these new boundary conditions, the cycle is completed by advancing the solid by $\Delta t$, which in practice is typically done by taking multiple, smaller time steps in the solid solver (i.e. subcycling) to effectively accommodate the more restrictive stability condition in the solid.

While the implementation of the described loosely coupling FSI methodology is straightforward with conventional solvers with consecutive time update, the utilization of the SAMR method in the fluid is non-apparent. In the VTF, we treat the 


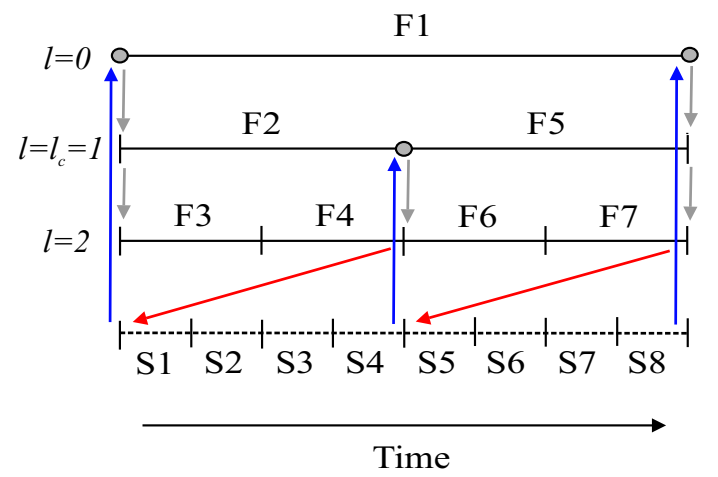

Figure 8: Data exchange between the recursive CFD solver and the linear thin-shell CSD solver throughout one SAMR root level time step.

fluid-solid interface as a discontinuity that is a-priori refined at least up to a coupling level $l_{c}$. The resolution at level $l_{c}$ has to be sufficiently fine to ensure an accurate wave transmission between fluid and structure, but will often not be the highest level of refinement. In order to incorporate the fluid-structure data exchange into the recursive SAMR algorithm, it has to be ensured that the updated mesh positions and nodal velocities are received before a regridding of the coupling level $l_{c}$ is initiated and that the hydrodynamic pressure loadings on the interface are evaluated after the highest available refinement level has reached the same discrete time as the updated level $l_{c}$. We visualize the data exchange between solid and SAMR fluid solver in Fig. 8 for an exemplary SAMR hierarchy with two additional levels with $r_{1,2}=2$. Figure 8 pictures the recursion in the SAMR method by numbering the fluid update steps (F) according to the order determined by the SAMR method. The order of the solid update steps $(\mathrm{S})$ on the other hand is strictly linear. The red arrows correspond to the sending of the interface pressures $p^{F}$ from fluid to solid at the end of each time step on level $l_{c}$. The blue arrows visualize the sending of the interface mesh and its nodal velocities $\boldsymbol{u}^{S}$ after each solid update. The modification of refinement meshes is indicated in Fig. 8 by the gray arrows; the initiating base level, that remains fixed throughout the regridding operation, is indicated by the gray circles.

In our current implementation, CFD and CSD solver are parallelized separately for distributed memory machines using independent rigorous domain decomposition methods. In order to facilitate an efficient communication of the distributed fluid-shell boundary information we have implemented a non-blocking high-level communication library that determines the necessary point-to-point communication patterns by intersecting Cartesian bounding boxes enclosing the local domains. Details on this communication library and also a detailed algorithmic description of the coupled SAMR method can be found in [17]. 


\section{Plate deformation from water hammer}

In order to validate the presented fluid-structure coupling technique, we consider an experimental setup developed by Deshpande et al. [18]. By firing a steel projectile on a piston inserted into the end of a water shock tube, a strong pressure wave is created that propagates through the water column and impinges onto a circular copper plate sealing the other end. The shock tube has a length of $1.3 \mathrm{~m}$ and a radius of $32 \mathrm{~mm}$. It is filled with water of density $\rho_{w}=1000 \mathrm{~kg} / \mathrm{m}^{3}$ at atmospheric pressure $p_{A}=101.3 \mathrm{kPa}$. At these conditions, the speed of sound in water is approximately $c_{w}=1482 \mathrm{~m} / \mathrm{s}$. The copper plate has a thickness of $0.25 \mathrm{~mm}$ and a radius of $r=$ $56 \mathrm{~mm}$, but is unconstrained only for $r<32 \mathrm{~mm}$. In our computations, the center of the plate is initially located at the coordinate origin and the shock tube middle axis is aligned with the positive x-axis. In all simulations, the piston has an initial velocity of $v_{0}=-22.94 \mathrm{~m} / \mathrm{s}$ and an averaged mass per unit area of $\bar{m}=74.1 \mathrm{~kg} / \mathrm{m}^{2}$.

Immediately after the wave impact on the target, the induced solid motion causes cavitation in the plate vicinity. We employ the stiffened equation of state sketched in Sec. 2.1 with parameters $\gamma=7.415$ and $p_{\infty}=296.2 \mathrm{MPa}$, but apply the correction

$$
E:=\frac{\gamma p_{\infty}}{\rho(\gamma-1)}+\frac{1}{2} \boldsymbol{u}^{T} \boldsymbol{u}, \quad \text { for } p<0
$$

after every fluid time step to set all negative hydrodynamic pressures to zero.

\subsection{Verification of loading conditions}

The motion of the piston creates a quasi one-dimensional pressure spike with exponential decay rate that propagates through the water column. We incorporate the piston movement into the computational setup by employing a separate signed distance level set function that corresponds to the piston boundary in contact with the fluid. The level set is initially positioned at $x=1.3 \mathrm{~m}$ and assumed to move with constant velocity $v_{0}$. During a simulation, we integrate the law of motion for the piston

$$
\bar{m} \dot{v}=-\left(\bar{p}-p_{A}\right)
$$

twice with the forward Euler method and update level set position and velocity $v$ in direction of the tube middle axis (to be used as wall normal velocity $w_{n}$ for this level set, cf. Sec. 2.3) in every time step. As the piston is constrained in all other directions, it suffices to consider the hydrodynamic pressure averaged across the piston boundary $\bar{p}$ and to use the averaged piston mass per unit area $m$.

By assuming the wave to propagate with constant velocity $c_{w}$, a traveling wave 

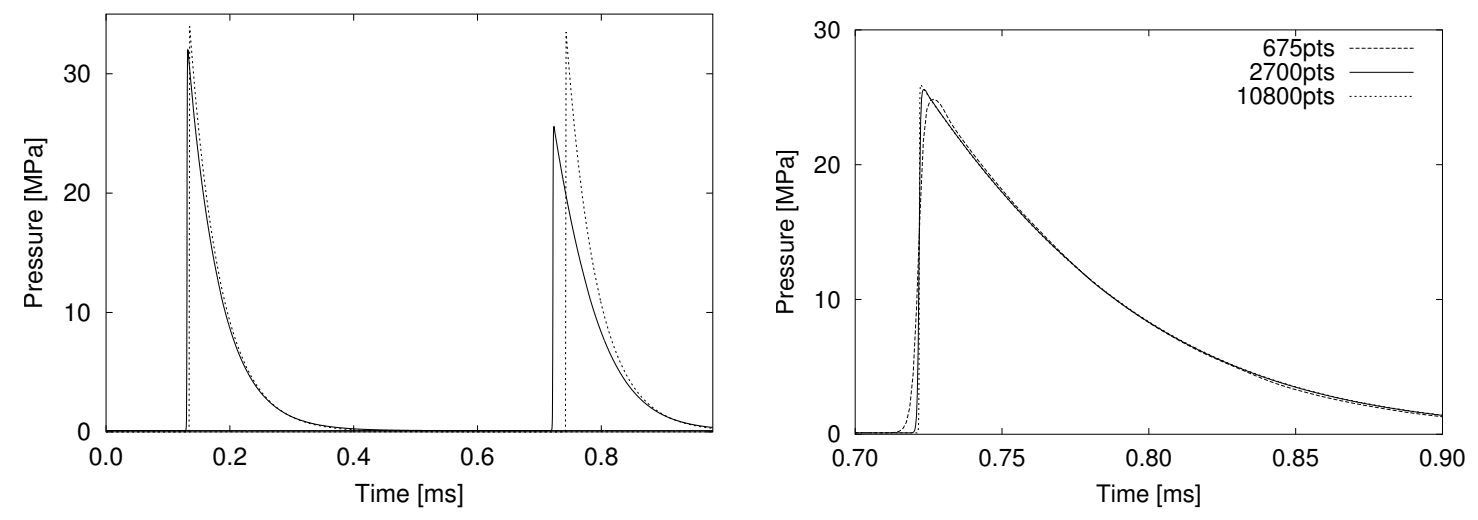

Figure 9: Left: comparison of the traveling wave approximation (18) (dotted) with computed pressure traces (solid) at $x=1.1 \mathrm{~m}$ (left) and $x=0.2 \mathrm{~m}$ (right). Right: mesh refinement study for computed pressure trace at $x=0.2 \mathrm{~m}$.

solution of the form

$$
p(x, t)= \begin{cases}\rho_{w} c_{w} v_{0} \exp \left(-\frac{t-x / c_{w}}{\bar{m} /\left(\rho_{w} c_{w}\right)}\right), & t \geq \frac{x}{c_{w}} \\ 0, & \text { otherwise }\end{cases}
$$

can be derived for the pressure evolution at a fixed spatial location that is found to be in reasonable agreement with experimental measurements close to the piston boundary [18]. The left plot of Fig. 9 compares (18) evaluated at the locations $x=1.1 \mathrm{~m}$ and $x=0.2 \mathrm{~m}$ with pressure traces derived from a one-dimensional unigrid finite volume simulation with 2700 cells on the domain $[0 \mathrm{~m}, 1.35 \mathrm{~m}]$. The agreement at early times is very good, verifying the correctness of our computational setup. At later times, close to the target, the simulation necessarily differs from the unaltered (non-dispersive) traveling wave, because our computational model correctly considers the rise in density in the compression wave and the resulting local change of speed of sound, cf. Eq. (4). The right graphic of Fig. 9 compares the pressure traces derived from differently refined simulations to determine the minmal fluid resolution in the FSI setup.

\subsection{Fluid-structure interaction simulation}

From fluid-structure interaction experiments by Deshpande et al. it is known that the thin copper plate deforms plastically for the loading conditions of Sec. 6.1. The plate bulges severely outwards, but does not rupture. Hence, we use a signed distance level set function to represent shock tube and plate geometry and avoid having to consider the air on the other side of the plate. For the evaluation of $p^{F}$, $p^{+} \equiv p_{A}$ is used. The mesh for the plate considers the mounting holes and uses 8896 triangles. For $r \geq 32 \mathrm{~mm}$, the plate is constrained in the normal direction. For 


$\begin{array}{ll}\text { Mass density } & \rho=8920 \mathrm{~kg} / \mathrm{m}^{3} \\ \text { Young's modulus } & E=130 \mathrm{GPa} \\ \text { Poisson's ratio } & \nu=0.31 \\ \text { Yield stress } & \sigma_{y}=38.5 \mathrm{MPa} \\ \text { Reference plastic strain } & \epsilon_{0}^{p}=0.0091 \\ \text { Hardening exponent } & 1 / n=0.627\end{array}$

Table 1: Material properties for annealed copper.
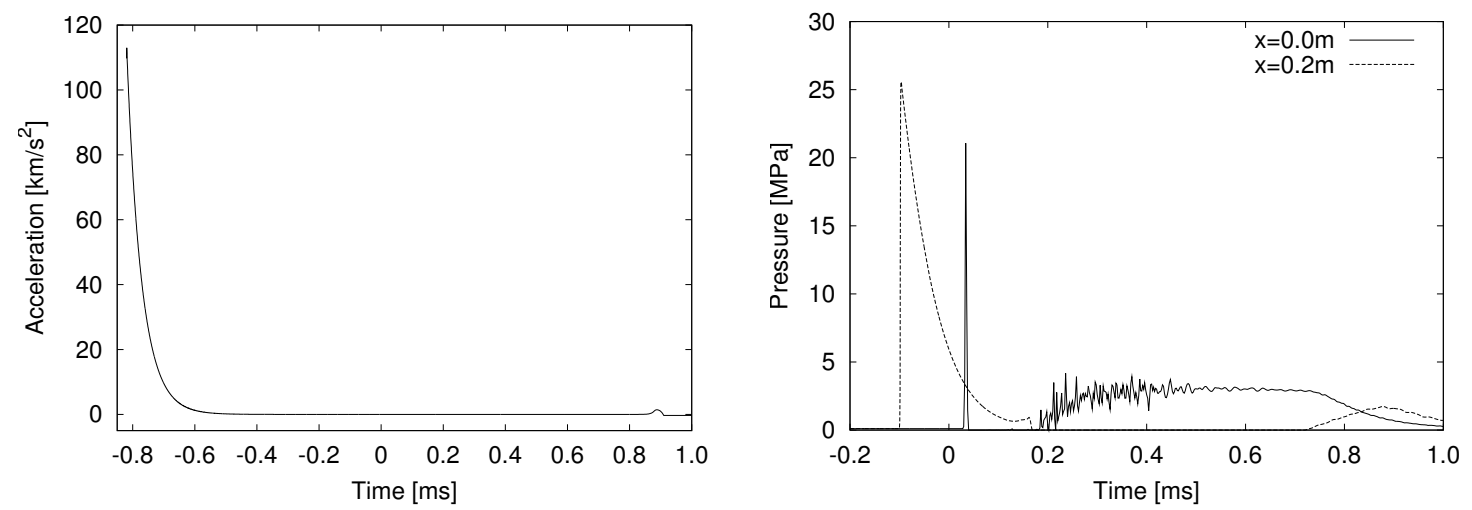

Figure 10: Left: computed piston acceleration for the entire simulation time. Right: fluid pressure traces at $x=0 \mathrm{~m}$ and $x=0.2 \mathrm{~m}$.

$r \geq 41 \mathrm{~mm}$, it is fully fixed. ${ }^{1}$ The material paramaters for the $J_{2}$ plasticity model and annealed copper are given in Table 1. It is assumed that the annealed copper is strain-rate insensitive and strain-softening effects have been not considered.

To ensure the correct boundary conditions throughout the whole simulation, the three-dimensional fluid domain covers with $[-0.05 \mathrm{~m}, 1.35 \mathrm{~m}] \times[-0.04 \mathrm{~m}, 0.04 \mathrm{~m}] \times$ $[-0.04 \mathrm{~m}, 0.04 \mathrm{~m}]$ the entire shock tube. The law of motion for the piston is still considered. The initial conditions for Eq. (17) in the FSI setup are the exact piston location and velocity at $t=0.82 \mathrm{~ms}$ from the one-dimensional simulation. The left graphic of Fig. 10 shows the computed piston acceleration for the entire simulated time. From now on, the time origin is set to the start of the FSI simulation which itself is initialized with the one-dimensional flowfield after simulating $0.82 \mathrm{~ms}$ physical time. At this time, the head of the pressure wave is close to $x=0.05 \mathrm{~m}$. The right graphic of Fig. 10 shows pressure traces in the FSI simulation in the tube middle axis at the locations $x=0.2 \mathrm{~m}$ and $x=0 \mathrm{~m}$. The impact of the pressure wave onto the plate at $t \approx 0.03 \mathrm{~ms}$ and the water cavitation immediately after can be clearly inferred. An expansion wave travels upstream through the water column

\footnotetext{
${ }^{1}$ Neglecting the mounting holes in the plate discretization does not alter the computational results. The presented simulation considers them only to allow the optical comparison of Fig. 12.
} 

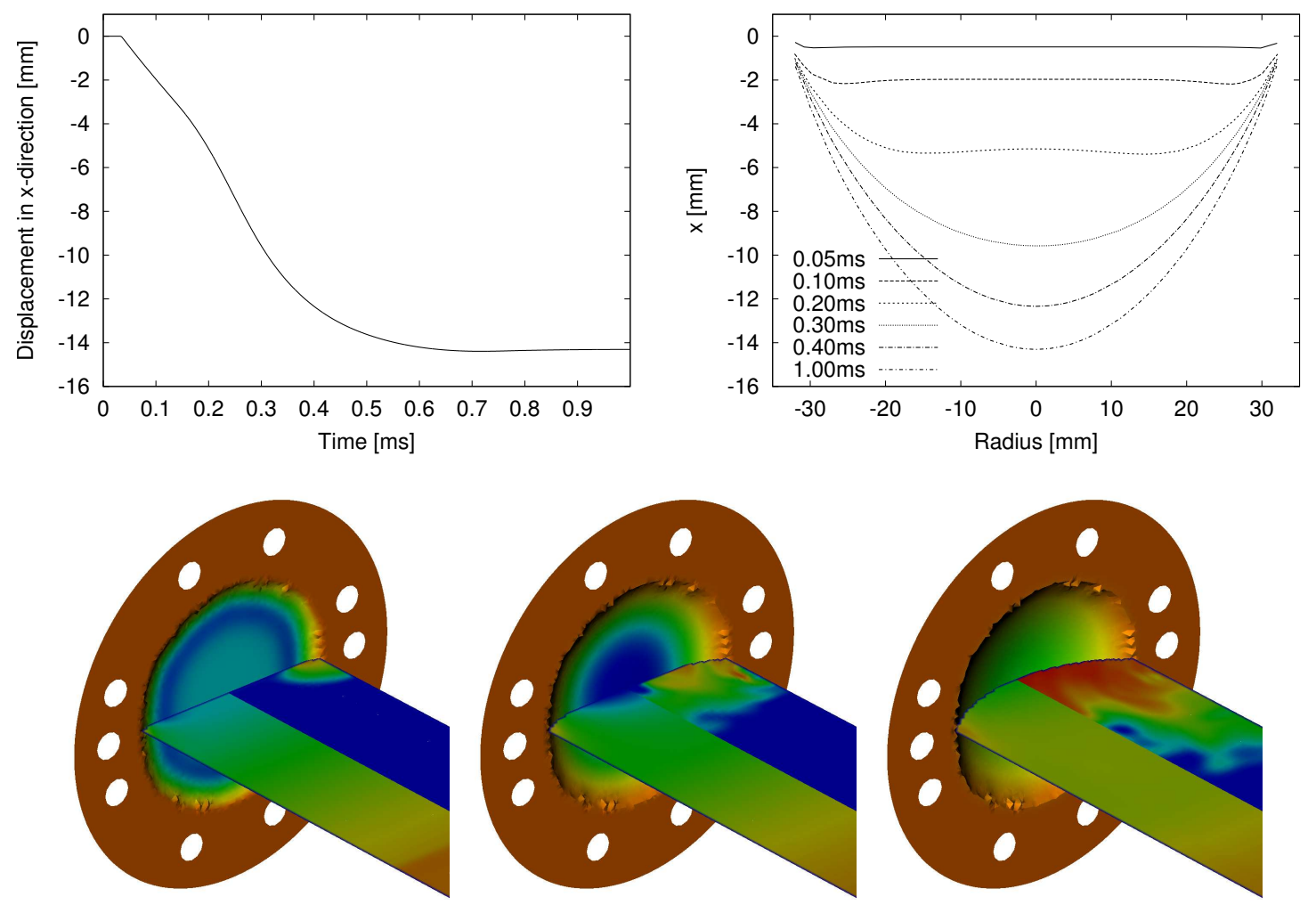

Figure 11: Upper row: maximum out-of-plane displacement of the plate versus time (left) and deflection history of the plate during the coupled simulation (right). Lower row: out-of-plane velocity of the plate and color plots of fluid pressure (upper half) and longitidunal fluid velocity at $t=0.2 \mathrm{~ms}, t=0.4 \mathrm{~ms}$, and $t=0.8 \mathrm{~ms}$ (from left to right).

and induces a small piston acceleration around $t=0.9 \mathrm{~ms}$.

To concentrate the computational resources for the fluid in the region of interest, the computation uses an SAMR base mesh of $350 \times 20 \times 20$ cells and two additional levels with refinement factors $r_{1,2}=2$. The fluid mesh is always fully refined along the plate boundary and at the front of the incoming pressure wave. The shock tube boundary is statically refined at level 2 for $x<0.206 \mathrm{~m}$ and at level 1 for $x<0.43 \mathrm{~m}$. The coupling level for the fluid-structure data exchange is naturally set to $l_{c}=2$.

The simulation was run on 8 nodes of a Intel-3.4 GHz-Xeon dual processor system connected with dual Gigabit Ethernet Interconnect (12 fluid and 4 solid processes) and required approximately $8.1 \mathrm{~h}$ wall clock time, which corresponds to $\sim 130 \mathrm{~h}$ CPU. 2000 coupled time steps were calculated with fixed step size to $t_{e}=1.0 \mathrm{~ms}$, where 5 solid solver sub-steps were taken during each fluid time step at the coupling level (cf. Sec. 5). The lower row of Fig. 11 shows a sequence of color graphics that visualize the fluid-structure interaction. The upper half of the color plane through 

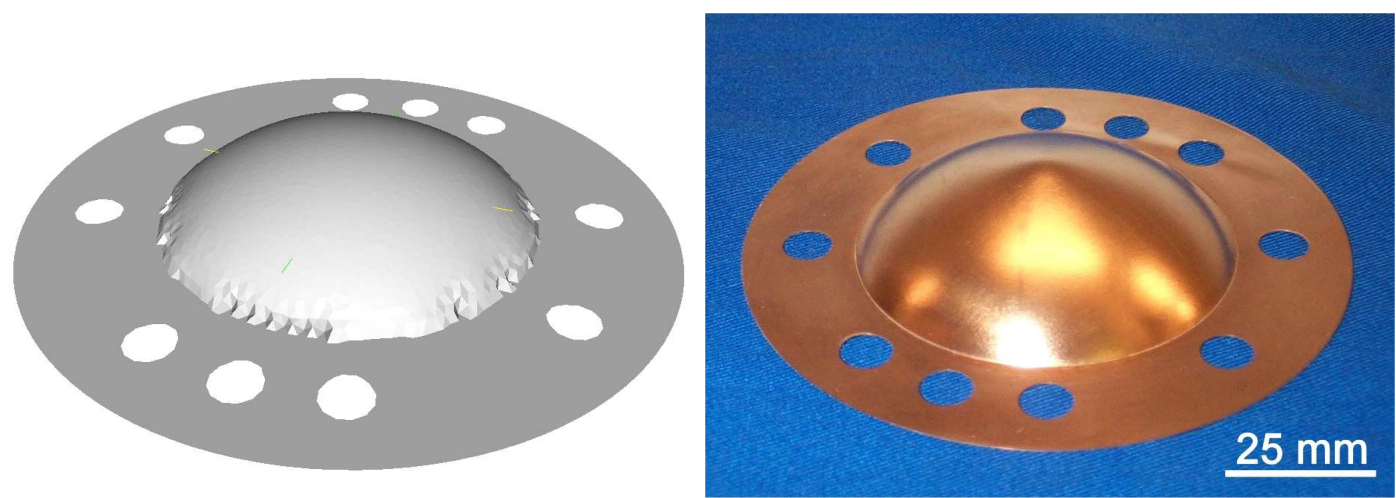

Figure 12: Deformed plate at end of simulation at $t_{e}=1.0 \mathrm{~ms}$ (left) and after the experiment (right).

the fluid domain shows the hydrodynamic pressure. The lower half and the coloring of the plate display the velocity component in x-direction. The upper row of Fig. 11 depicts the displacement of the plate center and the deflection of the plate middle plane.

After the initial pressure impulse, the fluid-structure interaction is apparently separated into two phases. During the first $0.2 \mathrm{~ms}$, the plate deformation occurs with constant velocity as the cavitating water does not transmit significant forces onto the plate. The deflection of the plate is characterized by the appearence of a stationary plastic hinge at the boundary and a second instationary plastic hinge traveling towards the plate center [22]. Shortly after, the fluid expansion wave leaves the plate boundary. A hydrodynamic pressure of $\sim 4 \mathrm{MPa}$ builds up again directly at the plate until it declines finally from $t \approx 0.76 \mathrm{~ms}$ on. During this phase, the deformation is nonlinear and the plate deflection is convex. The displacement reaches it maximum at $t \approx 0.76 \mathrm{~ms}$ and remains almost unaltered until the end of the simulation at $t_{e}=1.0 \mathrm{~ms}$. A visual comparison of the finally deformed solid mesh in the FSI simulation with a photograph of a target plate by Deshpande et al. is given in Fig. 12. The agreement is apparently very good. The obtained maximum deflection of $14.4 \mathrm{~mm}$ is also in good agreement with Qui et al.'s [36] analytic estimate of $16.1 \mathrm{~mm}$ (Equation 21a in [36]). Note that Qui et al.'s estimate is for an ideally plastic material. In contrast, the presented computations include a strain-hardening effect, which has a reducing effect on the maximum displacements. Further, Qui et al. base their analysis on the traveling wave (18) that overpredicts the pressure maximum exposed to the plate, see also left graphic of Fig. 9. 


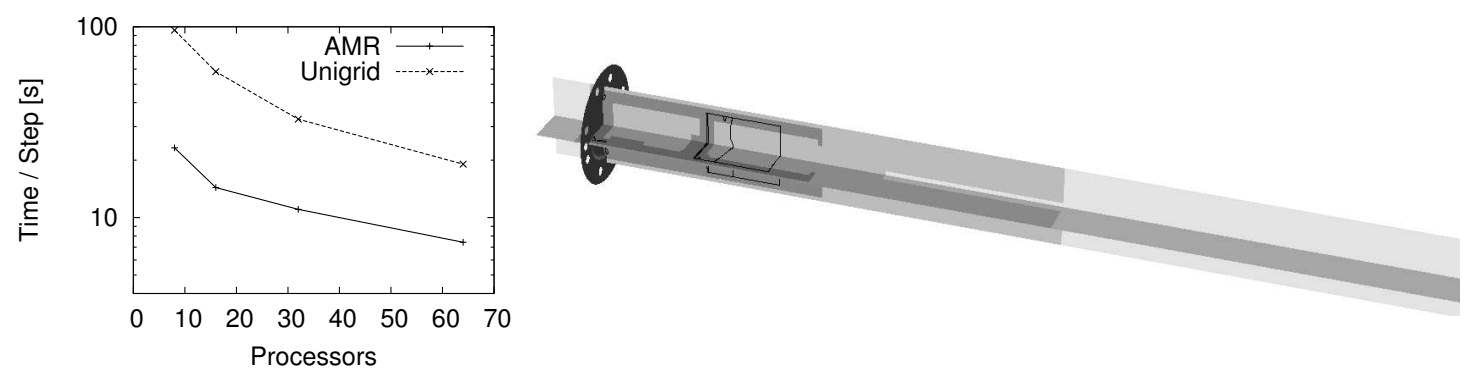

Figure 13: Strong scalability test for the first 200 fluid-structure coupling time steps for the simulation of Sec. 6.2 with fluid mesh adaptation and without (right). The left graphic visualizes the levels of block-structured mesh refinement in the fluid domain (shaded in gray tones) at $t=0.2 \mathrm{~ms}$.

\subsection{Computational performance}

In order to quantify the parallel performance of our current MPI-based implementation, we have performed a strong scalability study for the first 200 coupled time steps of the FSI simulation presented in the previous section. Further, we re-ran the test with a uniform fluid mesh of $1400 \times 80 \times 80$ cells to demonstrate the savings from dynamic fluid mesh adaptation. In every test computation, $1 / 4$ of the processors is dedicated to the thin-shell CSD solver, 3/4 to the CFD solver. Both test series were performed on the distributed memory machine used already in Sec. 6.2.

The left graphic of Fig. 13 compares the average wall clock times for a single coupled time step. A decrease in parallel efficiency due to a relative increase in costs for parallel data synchronization can be clearly inferred, but since both test series were run on a system with low-bandwidth interconnect, and not on a recent high-end machine, the timings provide most likely a lower bound for the parallel performance that users can expect from the VTF framework. The benefit from using dynamic mesh adaptation in the fluid is apparent. The right graphic shows a typical snapshot of the computational setup (showing only part of the fluid domain) that displays the regions of different refinement in gray tones. While $8.96 \mathrm{M}$ finite volume cells are necessary in the uniform case, the adaptive simulation uses only $\sim 1.2 \mathrm{M}$ cells on average, where the local resolution at the relevant solid structures and the pressure front remains the same.

\section{Detonation-driven tube deformation}

A multi-physics fluid-structure interaction configuration that is particularly challenging to simulate has been investigated experimentally by Chao [7]. The setup consists of a detonation tube of $1.52 \mathrm{~m}$ length to which thin-walled aluminum (Al6061T6) test tubes are attached. The test specimen have a length from $0.457 \mathrm{~m}$ to 

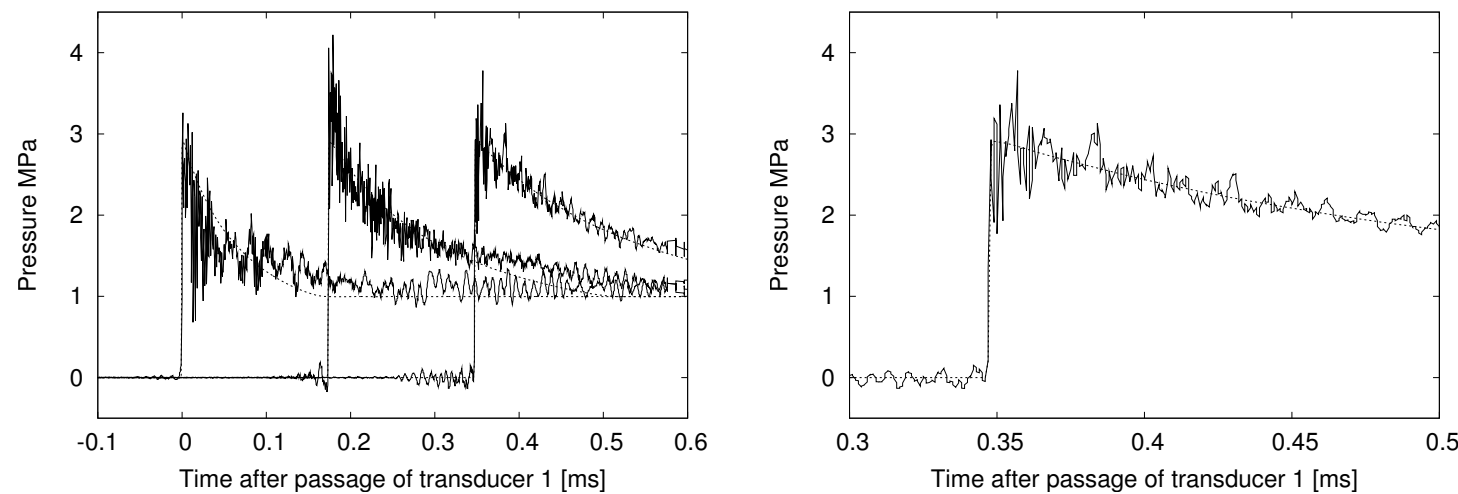

Figure 14: Left: comparison of the pressure traces at $x=0.38 \mathrm{~m}$ (transducer 1 ), $x=0.78 \mathrm{~m}, x=1.18 \mathrm{~m}$ (from left to right) in an experiment (solid) and in a $1 \mathrm{~d}$ simulation with the CV burn model (dotted). Right: enlargement for $x=1.18 \mathrm{~m}$.

$0.896 \mathrm{~m}$, an inner radius of $19.75 \mathrm{~mm}$, and a wall thickness of $0.89 \mathrm{~mm}$. While the lower end of the device is closed, a thin diaphragm seals the upper end. The entire apparatus is filled with a perfectly stirred combustible mixture of ethylene and oxygen at equivalence ratio $1\left(\mathrm{C}_{2} \mathrm{H}_{4}+3 \mathrm{O}_{2}\right)$ at room temperature $295 \mathrm{~K}$. The initial pressure varies from $p_{0}=80 \mathrm{kPa}$ to $p_{0}=180 \mathrm{kPa}$. The mixture is thermally ignited at the closed end and the combustion transitions quickly to a detonation wave. When it enters the test specimen, the detonation is close to the Chapman-Jouguet (CJ) limit of quasi-stationary self-sustained propagation. Its velocity is between $2300 \mathrm{~m} / \mathrm{s}$ and $2400 \mathrm{~m} / \mathrm{s}$ and the pressure values in the fully reacted Chapman-Jouguet state range between $p_{\mathrm{CJ}} \approx 2.60 \mathrm{MPa}$ and $p_{\mathrm{CJ}} \approx 6.10 \mathrm{MPa}$, depending on the initial pressure $p_{0}$. Since the lower end of the tube is closed, a rarefaction wave occurs immediately behind the detonation, which mandatorily needs to be considered in accurate numerical simulations. In all computations, we utilize a constant adiabatic mixture coefficient of $\gamma=1.24$, which is a good approximation to the value in the CJ state and a reasonable compromise between the constant value behind the rarefaction wave of $\sim 1.12$ and the value 1.4 in the air surrounding tube and specimen.

\subsection{Verification of detonation model and loading conditions}

In order to ensure the correct function of the detonation model described in Sec. 2.2, we carry out one-dimensional detonation simulations for an initial pressure of $p_{0}=$ $100 \mathrm{kPa}$. Separate calculations using Chapman-Jouguet theory (cf. [21]) that employ the full GRI 3.0 reaction mechanism predict a detonation velocity of $D_{\mathrm{CJ}} \approx$ $2376 \mathrm{~m} / \mathrm{s}$, but we set the only parameter of the constant-volume burn model, the heat release parameter $q$, to $q=4.70408 \mathrm{MJ} / \mathrm{kg}$ giving a detonation velocity of $D_{\mathrm{Ex}}=2291.7 \mathrm{~m} / \mathrm{s}$. This average speed of propagation has been measured for the particular experimental setup [7] we are interested in. We use a one-dimensional 
setup with a domain length of $2.15 \mathrm{~m}$ encompassing the detonation tube and the longest specimen, reflective wall boundary conditions at the lower end and zero gradient outflow conditions at the upper domain boundary. A base mesh of 1148 cells plus one additional level of dynamic refinement with factor $r_{1}=4$ is employed. The refinement criteria are simple scaled gradients of total density, pressure and mass fraction $Y$. In the computation, the detonation is initiated by setting the values in all cells with midpoint $<5 \mathrm{~mm}$ to the CJ values. Note that this is an unavoidable modeling idealization as the CJ limit is reached in the experiment only close to the test specimen.

Figure 14 gives a comparison of the temporal pressure traces at the locations $x=0.38 \mathrm{~m}$ (transducer 1 ), $x=0.78 \mathrm{~m}$, and $x=1.18 \mathrm{~m}$ in the one-dimensional simulation with experimentally measured pressure traces (time origins in both traces adjusted to $t=0$ when the detonation front reaches transducer 1 ). The agreement is very good considering the natural fluctuations in experimental measurements and the idealized computational initial conditions.

To verify the equivalence of the chosen detonation model to fully resolved detonation computations for the time scales relevant to us, a simulation has been undertaken utilizing the one-step Arrhenius reaction (6) instead of the CV burn model. Based on stationary calculations of the internal detonation structure according to the theory after Zel'dovich, von Neumann, and Döring (see [21] for a detailed account) using the detailed chemical kinetics of the GRI 3.0 mechanism, we set the activation energy to $E_{\mathrm{A}}=25,000 \mathrm{~J} / \mathrm{mol}$ and the frequency parameter to $k=2 \cdot 10^{7} \mathrm{~s}^{-1}$. As the utilization of Eq. (6) requires an extremely high local resolution in the reaction zone, this comparative computation uses an SAMR base mesh of 4000 cells and three additional levels with identical refinement factors $r_{1,2,3}=4$. Despite the use of mesh adaptation, the computation requires several hours CPU compared to only seconds in the previous case.

Figure 15 compares the pressure distributions of the one-dimensional computations with one-step Arrhenius reaction (6) and those derived with the CV burn model as the detonation propagates through the tube. Due to a reaction zone in the range of 0.01 to $0.1 \mathrm{~mm}$, the internal detonation structure, with its leading von Neumann pressure spike, appears as an isolated peak value. Only the enlargement on the right side of Fig. 15 reveals the internal detonation structure. With a short-term duration of less than a microsecond the spike has apparently negligible influence on the Taylor rarefaction wave following immediately behind. Since our succeeding FSI simulations involve fluid-structure interaction times of several hundred microseconds, it is physically justified to employ the CV burn model in the following for the hydrodynamic loading prediction.

In three space-dimensions, we ensure the correct consideration of the Taylor rarefaction wave by initializing the flow field with the data from corresponding one-dimensional simulations taken at the moment when the detonation enters the 

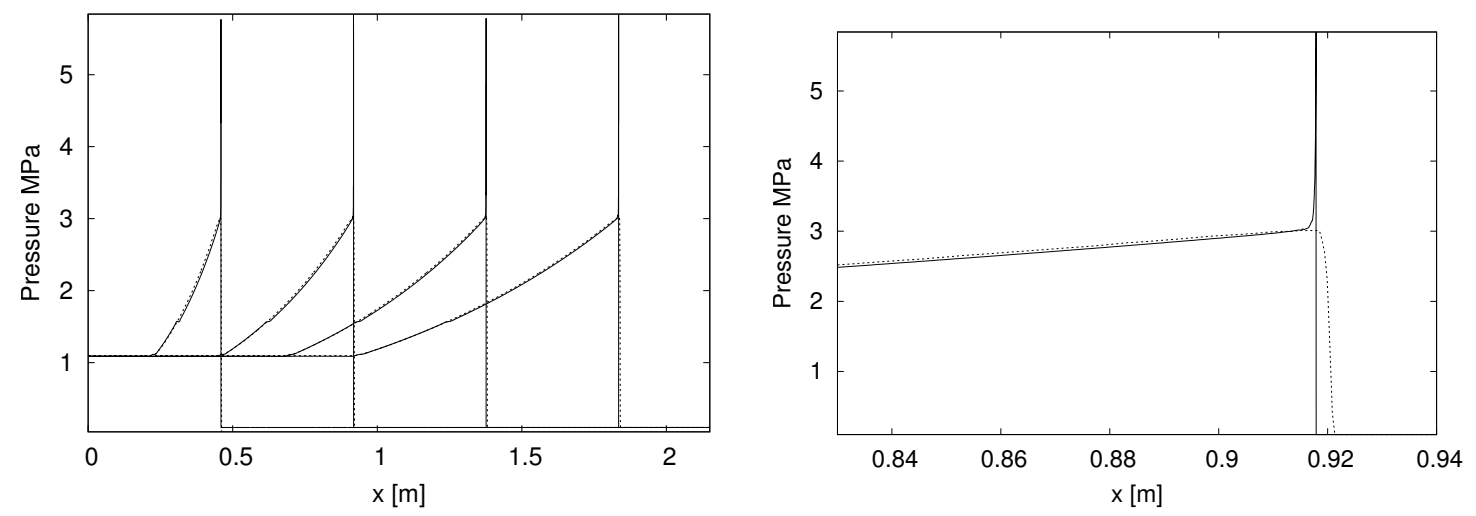

Figure 15: Left: comparison of pressure distribution for the one-step chemistry (solid) with the CV burn model (dotted) $0.2 \mathrm{~ms} 0.4 \mathrm{~ms}, 0.6 \mathrm{~ms}$, and $0.8 \mathrm{~ms}$ after ignition (time steps from left to right). Right: enlargement for $0.4 \mathrm{~ms}$.

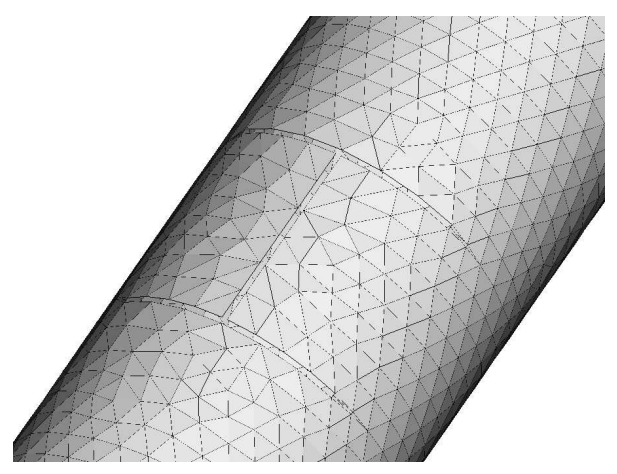

Figure 16: The finite element mesh for the thin-shell solver (in the reference configuration) used for the coupled flap opening simulation.

specimen. While only the test specimen is simulated in the CSD solver, the CFD solver considers an additional tubular domain $0.92 \mathrm{~m}$ upstream. The extension is modeled by prescribing the level set function for the embedded boundary method directly and its purpose is to ensure the correct inflow of the Taylor wave into the specimen.

\subsection{FSI simulation of detonation-driven venting}

As a qualitative validation test for large plastic material deformations, an experiment has been conducted in which an " $H$ " shape pattern is cut into a specimen of $0.896 \mathrm{~m}$ length. Each cut is $25 \mathrm{~mm}$ long (see Figure 16). One cut is in the longitudinal direction with its midpoint $0.444 \mathrm{~m}$ away from the inlet. The two other cuts extend perpendicular into the circumferential direction. The combustible mixture is the same as in Sec. 7.1. When the detonation wave passes the pre-flawed region, the 

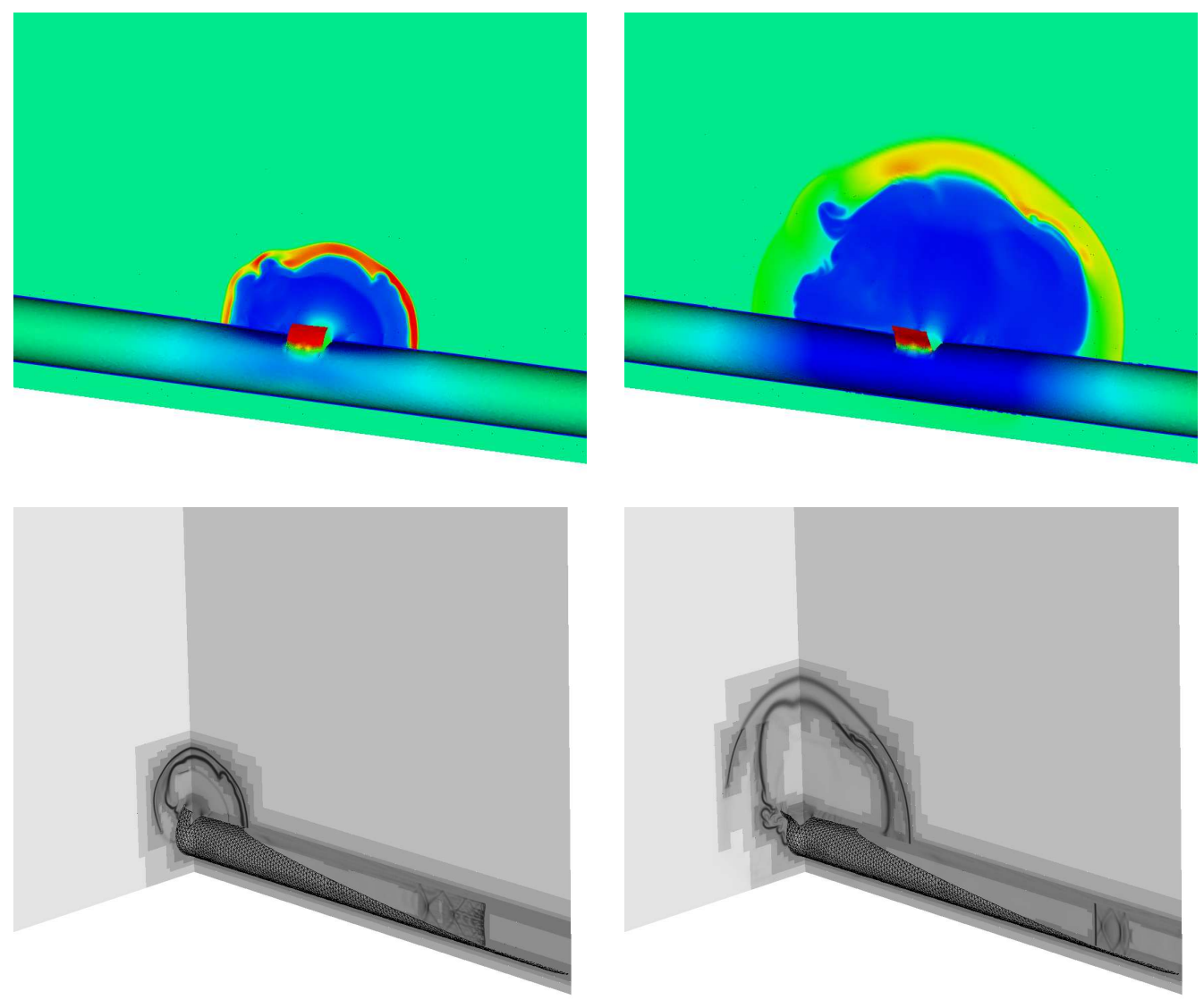

Figure 17: Upper row: color plot of fluid density in plane perpendicular to z-axis and shell displacements in the z-direction $t=0.092 \mathrm{~ms}$ (left) and $t=0.212 \mathrm{~ms}$ (right) after the detonation front has reached the middle of the longitudinal slot. Lower row: schlieren plot of fluid density in planes perpendicular to $\mathrm{x}$ - and z-axis on different refinement levels (gray).

two flaps open up and the high pressure in the Taylor wave causes a venting of the combustion products into the air. To allow for an undisturbed leakage, we use a relative large computational domain of $[-0.920 \mathrm{~m}, 0.896 \mathrm{~m}] \times[-0.0375 \mathrm{~m}, 0.5625 \mathrm{~m}] \times$ $[-0.390 \mathrm{~m}, 0.390 \mathrm{~m}]$, where the beginning of the specimen is now set to $x=0$. The opening of the flaps and the gaseous venting are visualized in the upper row of Fig. 17.

In this computation, an SAMR base mesh of $242 \times 80 \times 104$ cells with 3 additional level and refinement factors $r_{1,2}=2$ and $r_{3}=4$ is used. Additional to the refinement criteria in Sec. 7.1, that capture the detonation front reliably at the highest level, the walls of the test specimen are always fully refined. The effective resolution at the walls allows for an offset parameter of $h=0.81 \mathrm{~mm}$, which is less than two times 


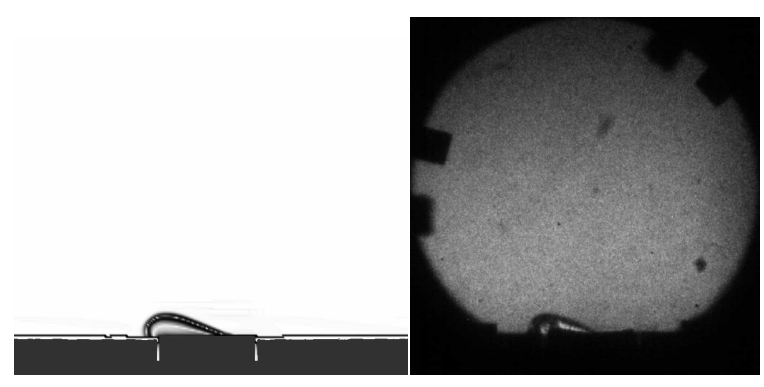

$0.002 \mathrm{~ms}$

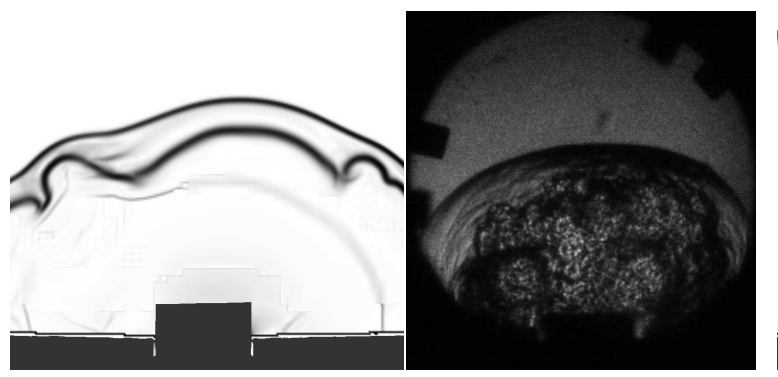

$0.092 \mathrm{~ms}$
$0.000 \mathrm{~ms}$

$0.090 \mathrm{~ms}$

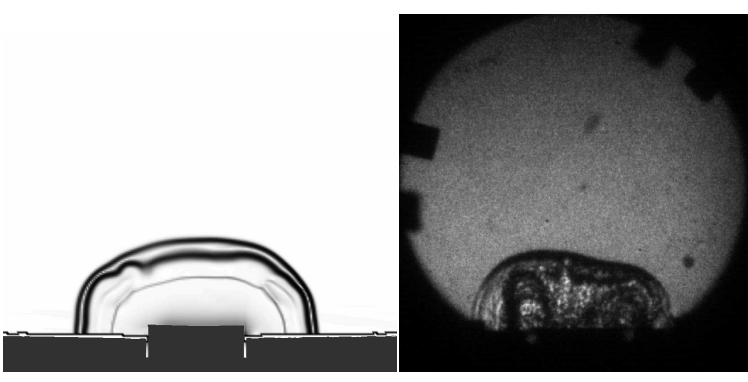

$0.032 \mathrm{~ms}$

$0.030 \mathrm{~ms}$

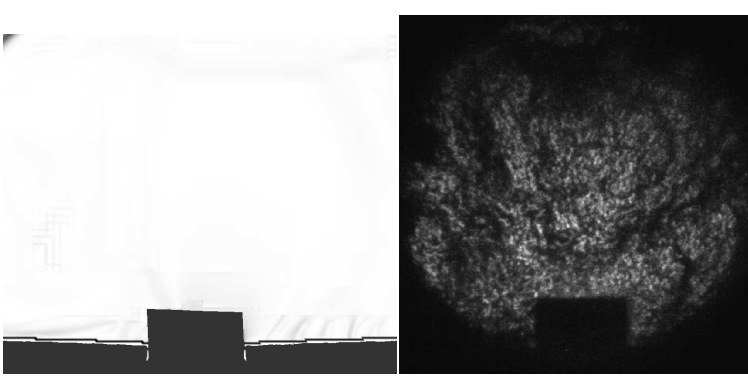

$0.212 \mathrm{~ms}$

$0.210 \mathrm{~ms}$

Figure 18: Simulated schlieren pictures of fluid density and side view of the deforming solid mesh compared to schlieren photographs taken in a corresponding experiment.

the exact wall thickness of $0.445 \mathrm{~mm}$. The maximal refinement is limited for $x<0$, $y>0.15 \mathrm{~m}$ and $|z|>0.1575 \mathrm{~m}$. The dynamic evolution of the block-structured mesh hierarchy is depicted in the lower row of Fig. 17. The graphics show schlieren images of the fluid density on the three refinement levels, which are visualized by shading their domains in different gray tones. The graphics highlight the enormous efficiency gain from dynamic mesh adaptation. An equivalent unigrid CFD calculation would require $>7.9 \cdot 10^{9}$ cells, but our SAMR computation uses only $\sim 4.0 \cdot 10^{7}$ cells on average.

For the CSD sub-problem, we use a triangular input mesh of 17,056 elements, in which each base element is subdivided internally into four smaller elements. The initial mesh is shown in Fig. 16. We employ a $J_{2}$ plasticity model for aluminum with power-law hardening and thermal softening as bulk material model [12]. The parameters for our particular model given in Table 2 have been adapted from the Johnson-Cook material parameters reported by Lesuer et al. [23]. An alternative reference for $\mathrm{Al}$ 6061-T6 material parameters is Warren et al. [41].

The computation ran on 72 Opteron-2.2 GHz processors connected with Infiniband network for about $60 \mathrm{~h}$ wall clock time $(\sim 4300 \mathrm{~h} \mathrm{CPU})$ to a final time of $t_{e}=0.460 \mathrm{~ms} .460$ coupled time steps with fixed step size have been simulated with 5 solid solver sub-steps in each fluid time step at the coupling level. As the wall 


$\begin{array}{ll}\text { Mass density } & \rho=2719 \mathrm{~kg} / \mathrm{m}^{3} \\ \text { Young's modulus } & E=69.0 \mathrm{GPa} \\ \text { Poisson's ratio } & \nu=0.33 \\ \text { Yield stress } & \sigma_{y}=275 \mathrm{MPa} \\ \text { Reference plastic strain } & \epsilon_{0}^{p}=0.001 \\ \text { Hardening exponent } & 1 / n=0.07 \\ \text { Reference plastic strain rate } & 0.61 / \mathrm{s} \\ \text { Strain rate sensitivity exponent } & 1 / m=0.01\end{array}$

Table 2: Material properties for Al 6061-T6.

boundary is refined up to the highest level, the coupling level is set to $l_{c}=3$. In Fig. 18, a series of schlieren photographs from the experiment showing the fluid venting and flap opening are compared to corresponding simulated images at a nearby time. The computational graphics display schlieren of the fluid density in the plane perpendicular to the z-axis together with a side view of the deforming solid mesh. The time origin is set to the moment when the detonation passes the middle of the longitudinal slot. The agreement in flow evolution and solid deformation is reasonable confirming the appropriateness of the chosen computational setup.

\subsection{FSI simulation of detonation-driven fracture}

Finally, we present one exemplary fluid-structure interaction computation that involves the rupture of the test specimen. The initial pressure is $p_{0}=180 \mathrm{kPa}$ and the specimen has a length of $0.457 \mathrm{~m}$. To ensure a reproducible fracture pattern, the specimen has a central longitudinal notch of $63.2 \mathrm{~mm}$ parallel to the middle axis, which is modeled as an initial crack in the computations. The computational setting is similar as described above, but no fluid mesh adaptation has been employed yet: The simulation has preliminary character.

The material model for the cohesive interface elements is a linearly decreasing envelope with a plane stress fracture toughness $K_{\mathrm{IC}}=30 \mathrm{MPa} \sqrt{m}$ [10]. In accordance with $\mathrm{Li}$ et al.'s [25] numerical computations of thin-sheet ductile fracture, the crack initiation stress is chosen to be $\sigma_{c}=2 \sigma_{y}$, where $\sigma_{y}$ is the yield stress of the bulk material.

Figure 19 visualizes the results for a shell mesh of 8665 elements and a uniform Cartesian fluid mesh of $725 \times 40 \times 40$ cells that required $\sim 900 \mathrm{~h} \mathrm{CPU}$ and ran for about $16.5 \mathrm{~h}$ wall clock time on 27 nodes of a Pentium-4-2.4 GHz dual processor system (21 fluid and 33 solid processes). 1300 coupled time steps with fixed step size to a final time of $t_{e}=0.260 \mathrm{~ms}$ have been calculated (20 solid solver sub-steps in each fluid time step). The upper graphic of Fig. 19 shows the beginning of the 


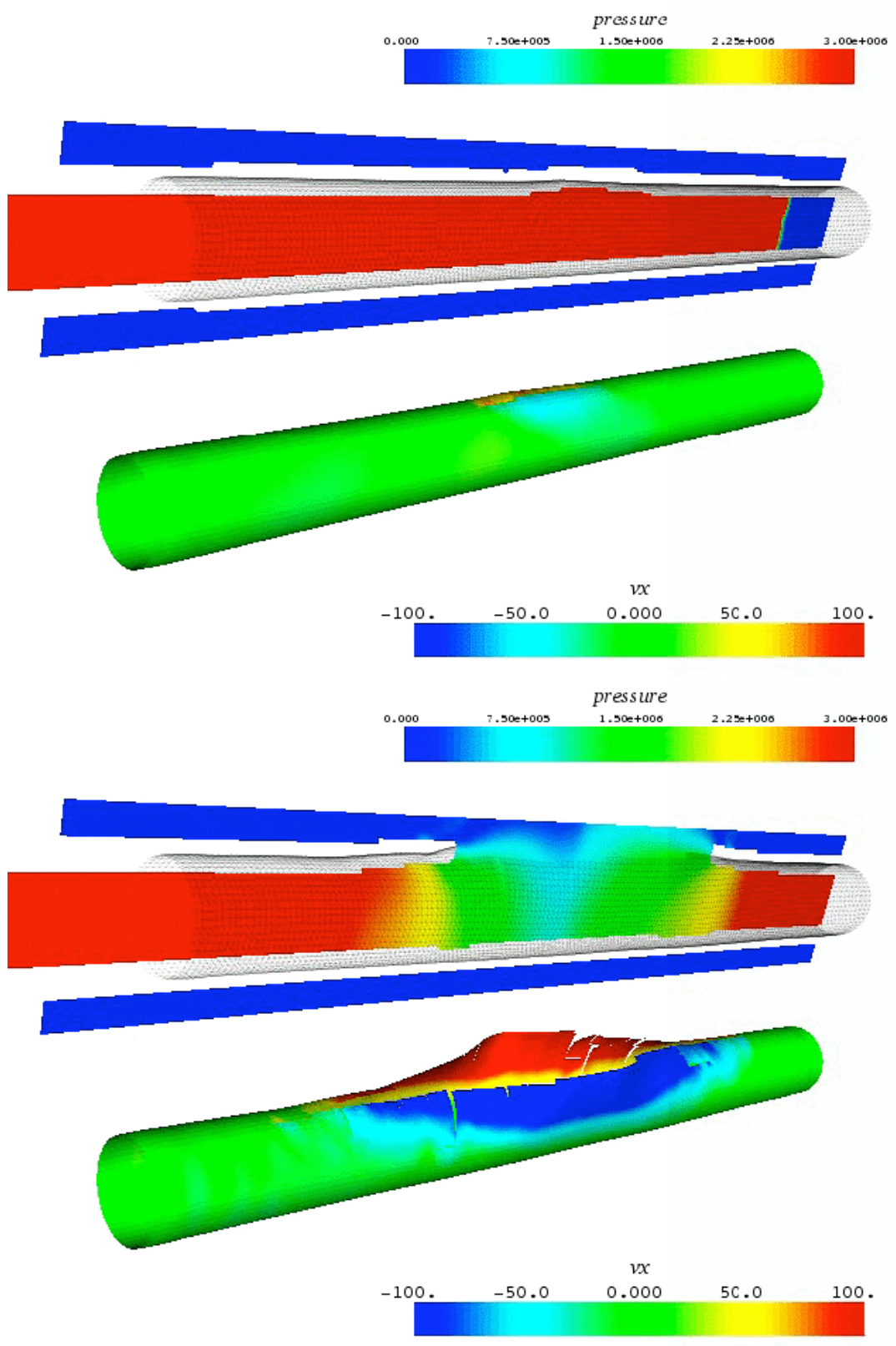

Figure 19: Coupled simulation of detonation-driven rupture of a thin aluminum tube. Snapshots $t \approx 0.150 \mathrm{~ms}$ (upper graphic) and $t \approx 0.260 \mathrm{~ms}$ (lower graphic) after the detonation has passed the initial notch show the fracturing tube with solid velocity iso-contours on the solid surface mesh; corresponding cuts through the fluid domain visualize the resulting hydrodynamic venting. 
crack opening $\sim 0.150 \mathrm{~ms}$ after the detonation has passed the initial crack. The lower snapshot shows the rupture at the final time $0.260 \mathrm{~ms}$. The venting of high pressurized reacted gas from the opening slit and the cracking of the material are clearly visible. It is worth pointing out that during this simulation, the dynamic level set evaluation with the algorithm sketched in Sec. 4 and the update with the core Cartesian finite volume scheme have about the same computational costs on each fluid processor. This result confirms that our approach utilizing distance functions for implicit geometry representation is suitable for computing even complex FSI problems with large deformations and evolutions in the mesh topology with high computational efficiency.

\section{Conclusions}

A loosely coupled level-set-based fluid-structure coupling methodology for the timeaccurate simulation of thin flexible shells responding dynamically to supersonic shock waves has been described. The approach has been demonstrated to handle arbitrarily evolving thin-shells surrounded by fluid without problems. Two different fluidstructure interaction configurations have been investigated in detail to verify and validate the approach. In the first setup, the plastic deformation of a thin copper plate due to a piston-induced sonic pressure wave in water is predicted in excellent agreement with experimental observations. In the second configuration, the opening of flaps cut into a thin aluminum tube due to a supersonic gaseous detonation wave and the resulting hydrodynamic venting are found to be in reasonable agreement with experimental results. In particular, we have detailed the verification of the initially one-dimensional hydrodynamic loading conditions, whose accurate modeling is essential for the instationary high speed fluid-structure interaction problems discussed. As enabling components for high computational efficiency, we have highlighted dynamic mesh adaptation in the fluid sub-solver, an effective distance function evaluation algorithm and the parallel performance on typical Linux-PC clusters with distributed memory. The integrated implementation of these components in the software framework "Virtual Test Facility" is freely available for research purposes (cf. http://www.cacr.caltech.edu/asc).

\section{Acknowledgements}

This work has been carried out largely when all of the authors were at the ASC Center for the Dynamic Response of Materials the California Institute of Technology and were supported by the ASC program of the Department of Energy under subcontract no. B341492 of DoE contract W-7405-ENG-48. We thank particular D. I. Meiron for his continued interest and support. 
Part of this work was also sponsored by the Mathematical, Information, and Computational Sciences Division; Office of Advanced Scientific Computing Research; U.S. Department of Energy and was performed at the Oak Ridge National Laboratory, which is managed by UT-Battelle, LLC under Contract No. De-AC0500OR22725.

The authors would like to thank J. E. Shepherd and his group (J. C. Krok, Z. Liang, J. Karnesky and F. Pintgen) from the Graduate Aeronautical Laboratory of the California Institute of Technology for kindly providing the unpublished experimental validation results shown in the Figs. 14 and 18. Further, we would like to thank V. S. Deshpande from the Department of Engineering of the University of Cambridge for providing the validation result shown in Fig. 12.

\section{References}

[1] M. Aivazis, W. Goddard, D. I. Meiron, M. Ortiz M, J. Pool, J. Shepherd. A virtual test facility for simulating the dynamic response of materials. Comput. Science \& Engineering 2(2):42-53, (2000).

[2] P. M. A. Areias, J.-H. Song, T. Belytschko. Analysis of fracture in thin shells by overlapping paired elements. Computer Methods in Appl. Mechanics and Engineering, 195:5343-5360, (2006).

[3] M. Arienti, P. Hung, E. Morano, J. Shepherd. A level set approach to EulerianLagrangian coupling. J. Comput. Phys. 185:213-251, (2003).

[4] M. Berger and P. Colella. Local adaptive mesh refinement for shock hydrodynamics. J. Comput. Phys., 82:64-84, (1988).

[5] M. J. Berger and C. Helzel. Grid aligned h-box methods for conservation laws in complex geometries. In Proc. 3rd Intl. Symp. Finite Volumes for Complex Applications, Porquerolles, June (2002).

[6] D. Chapelle and K. J. Bathe. The Finite Element Analysis of Shells - Fundamentals. Springer, (2003).

[7] T.-W. Chao. Gaseous detonation-driven fracture of tubes. PhD thesis, California Institute of Technology, (2004).

[8] F. Cirak, M. Ortiz, P. Schröder. Subdivision surfaces: a new paradigm for thinshell finite-element analysis. Int. J. Numer. Meth. Engineering, 47:2039-2072, (2000). 
[9] F. Cirak and M. Ortiz. Fully $C^{1}$-conforming subdivision elements for finite deformation thin-shell analysis Int. J. Numer. Meth. Engineering, 51:813-833, (2001).

[10] F. Cirak, M. Ortiz, and A. Pandolfi. A Cohesive Approach to Thin-Shell Fracture and Fragmentation. Computer Methods in Appl. Mechanics and Engineering, 194:2604-2618, (2005).

[11] F. Cirak and R. Radovitzky. A Lagrangian-Eulerian Shell-Fluid Coupling Algorithm Based on Level Sets. Computers \&f Structures, 83:491-498, (2005).

[12] A. Cuitino and M. Ortiz. A material-independent method for extending stress update algorithms from small-strain plasticity to finite plasticity with multiplicative kinematics. Engineering Computations, 9:437-451, (1992).

[13] R. deBorst. The zero-normal stress condition in plane-stress shell elastoplasticity. Comm. Meth. Appl. Numer. Meth., 7:29-33, (1991).

[14] R. Deiterding. AMROC-Blockstructured Adaptive Mesh Refinement in Objectoriented $C++$. http://amroc.sourceforge.net.

[15] R. Deiterding. Parallel adaptive simulation of multi-dimensional detonation structures. PhD thesis, Brandenburgische Technische Universität Cottbus, (2003). Available at http://www.cacr.caltech.edu/ ralf/html/pub.htm.

[16] R. Deiterding. Construction and application of an AMR algorithm for distributed memory computers. In T. Plewa, T. Linde, and V. G. Weirs, editors, Adaptive Mesh Refinement - Theory and Applications, volume 41 of Lecture Notes in Computational Science and Engineering, pages 361-372. Springer, (2005).

[17] R. Deiterding, R. Radovitzky, S. P. Mauch, L. Noels, J. C. Cummings, and D. I. Meiron. A virtual test facility for the efficient simulation of solid materials under high energy shock-wave loading. Engineering with Computers, (2006). In press.

[18] V. S. Deshpande, A. Heaver, and N. A. Fleck. An underwater shock simulator. Royal Society of London Proceedings Series A 462(2067):1021-1041, (2006).

[19] R. P. Fedkiw. Coupling an Eulerian fluid calculation to a Lagrangian solid calculation with the ghost fluid method. J. Comput. Phys. 175:200-224, (2002).

[20] R. P. Fedkiw, T. Aslam, B. Merriman, and S. Osher. A non-oscillatory Eulerian approach to interfaces in multimaterial flows (the ghost fluid method). $J$. Comput. Phys., 152:457-492, (1999). 
[21] W. Fickett and W. C. Davis. Detonation. University of California Press, Berkeley and Los Angeles, California, (1979).

[22] N. Jones. Structural Impact. Cambridge University Press, (1989).

[23] D.R. Lesuer, G.J. Kay, and M.M. LeBlanc. Modeling large-strain, high rate deformation in metals. UCRL-JC-134118, Lawrence Livermore National Laboratory, (2001).

[24] R. J. LeVeque. Finite volume methods for hyperbolic problems. Cambridge University Press, New York, (2002).

[25] W. Li and T. Siegmund. An analysis of crack growth in thin-sheet metal via a cohesive zone model. Engineering Fracture Mechanics 69:2073-2093, (2002).

[26] R. Löhner, J. Baum, C. Charman, D. Pelessone. Fluid-structure interaction simulations using parallel computers. Lecture Notes in Computer Science 2565, Springer, Berlin, pp. 3-23, (2003).

[27] R. Löhner, J. Cebral, C. Yang, J. Baum, E. Mestreau, C. Charman, D. Pelessone D. Large-scale fluid-structure interaction simulations. Comput. Science 65 Engineering 6(3):27-37, (2004).

[28] C. L. Mader. Numerical modeling of detonations. University of California Press, Berkeley and Los Angeles, California, (1979).

[29] S. P. Mauch. Efficient Algorithms for Solving Static Hamilton-Jacobi Equations. PhD thesis, California Institute of Technology, (2003).

[30] S. P. Mauch, D. I. Meiron, R. Radovitzky, R. Samtaney. Coupled EulerianLagrangian simulations using a level set method. In Bathe K (ed). 2nd M.I.T. Conference on Computational Fluid and Solid Mechanics, Cambridge, MA, June 17-20, (2003).

[31] A. Needleman. A continuum model for void nucleation by inclusion debonding. J. Applied Mechanics 54:525-531, (1987).

[32] E. Onate, F. G. Flores. Advances in the formulation of the rotation-free basic shell triangle. Computer Methods in Appl. Mechanics and Engineering, 194:2406-2443, (2005).

[33] M. Ortiz, A. Pandolfi. Finite-deformation irreversible cohesive elements for three-dimensional crack-propagation analysis. Int. J. Numer. Meth. Engineering, 44:1267-1282, (1999). 
[34] M. Parashar, J. C. Browne. On Partitioning Dynamic Adaptive Grid Hierarchies. In Proc. of the 29th Annual Hawaii Int. Conf. on System Sciences, (1996).

[35] R. Phaal, C. R. Calladine. A simple class of finite elements for plate and shell problems. II: An element for thin shells, with only translational degrees of freedom. Int. J. Numer. Meth. Engineering, 35:979-996, (2005).

[36] X. Qui, V. S. Deshpande, N.A. Fleck. Dynamic Response of a Clamped Circular Sandwich Plate Subject to Shock Loading. Int. J. Numer. Meth. Engineering, 71:637-645, (2004).

[37] J. J. Quirk. An alternative to unstructured grids for computing gas dynamics flows around arbitrarily complex two-dimensional bodies. Computers Fluids, 23:125-142, (1994).

[38] U. Specht. Numerische Simulation mechanischer Wellen an Fluid-FestkörperMediengrenzen. Fortsch.-Ber. VDI Reihe 7 Nr. 398, VDU Verlag, Düsseldorf, (2000).

[39] E. F. Toro. Riemann solvers and numerical methods for fluid dynamics. Springer-Verlag, Berlin, Heidelberg, 2nd edition, (1999).

[40] The Virtual Test Facility. http://www.cacr.caltech.edu/asc. California Institute of Technology.

[41] T.L. Warren and M.J. Forrestal. Effects of strain hardening and strain-rate sensitivity on the penetration of aluminum targets with spherical-nosed rods. Int. J. Solids Structures, 35:3737-3753, (1998). 